\author{
Mar/2020 \\ Working Paper 20-09 \\ rcea.org/RePEc/pdf/wp20-09.pdf
}

\title{
SIGN RESTRICTIONS IN HIGH-DIMENSIONAL VECTOR AUTOREGRESSIONS
}

\author{
Dimitris Korobilis \\ University of Glasgow, UK \\ RCEA
}

Copyright belongs to the author. Short sections of the text, not exceeding three paragraphs, can be used provided proper acknowledgement is given.

The Rimini Centre for Economic Analysis (RCEA) was established in March 2007. RCEA is a private, nonprofit organization dedicated to independent research in Applied and Theoretical Economics and related fields. RCEA organizes seminars and workshops, sponsors a general interest journal, the Review of Economic Analysis (REA), and organizes a biennial conference, the Rimini Conference in Economics and Finance (RCEF). Scientific work contributed by the RCEA Scholars is published in the RCEA Working Paper series.

The views expressed in this paper are those of the authors. No responsibility for them should be attributed to the Rimini Centre for Economic Analysis. 


\title{
Sign restrictions in high-dimensional vector autoregressions
}

\author{
Dimitris Korobilis* \\ University of Glasgow
}

March 18, 2020

\begin{abstract}
This paper proposes a new Bayesian sampling scheme for VAR inference using sign restrictions. We build on a factor model decomposition of the reduced-form VAR disturbances, which are assumed to be driven by a few fundamental factors/shocks. The outcome is a computationally efficient algorithm that allows to jointly sample VAR parameters as well as decompositions of the covariance matrix satisfying desired sign restrictions. Using artificial and real data we show that the new algorithm works well and is multiple times more efficient than existing accept/reject algorithms for sign restrictions.
\end{abstract}

Keywords: high-dimensional inference; Structural VAR; Markov chain Monte Carlo; set identification

JEL Classification: C11, C13, C15, C22, C52, C53, C61

This paper is partial outcome of work I undertook as external expert for the Monetary Analysis Division of the European Central Bank (ECB). The views expressed in the paper are solely mine (as are any remaining errors) and they do not necessarily reflect views of the ECB or the Eurosystem.

I would like to thank Fabio Canova, Davide Pettenuzzo, as well as numerous seminar and conference participants for useful discussions and comments.

${ }^{*}$ Correspondence: Professor of Econometrics, Department of Economics, University of Glasgow, 40 University Avenue, Glasgow, G12 8QQ, UK; email:dikorobilis@googlemail.com 


\section{Introduction}

This paper proposes a new Bayesian Markov chain Monte Carlo (MCMC) algorithm for joint estimation of parameters of reduced-form vector autoregressions (VARs) and associated sign restrictions for structural identification. The main idea is to allow the reduced-form VAR disturbances to have a static factor model structure. By doing so, we show that we can directly impose sign (and other) restrictions on the covariance matrix that can be incorporated into parametric prior distributions; structural shocks are identical to factors, that is, they become latent quantities (parameters) that we estimate; and we can directly use the VAR likelihood to test the plausibility of identification restrictions. The specific factor formulation of the VAR disturbance terms allows to derive a simple and highly efficient Gibbs sampling scheme, where Monte Carlo samples from the posterior of sign restricted matrices are always accepted. Therefore, the main contribution of this paper is to establish a comprehensive methodology for estimation and identification in VARs that is computationally efficient, to the extent that it can be used with an arbitrarily large number of endogenous variables and/or shocks.

Our starting point is the following structural vector autoregression

$$
\mathbf{A y}_{t}=\mathbf{B} \mathbf{x}_{t}+\mathbf{u}_{t}
$$

where $\mathbf{y}_{t}$ is a $(n \times 1)$ vector of observed variables, $\mathbf{A}$ an $(n \times n)$ matrix summarizing their contemporaneous structural relations, $\mathbf{x}_{t}=\left(1, \mathbf{y}_{t-1}^{\prime}, \ldots, \mathbf{y}_{t-p}^{\prime}\right)^{\prime}$ a $(k \times 1)$ vector (with $k=$ $n p+1)$ containing a constant and $p$ lags of $\mathbf{y}, \mathbf{B}$ is an $(n \times k)$ matrix of coefficients, and $\mathbf{u}_{t}$ a $(n \times 1)$ vector of structural disturbances assumed to be $N\left(\mathbf{0}_{n \times 1}, \mathbf{D}\right)$ and uncorrelated both over time (white noise) and over the cross-section ( $\mathbf{D}$ is diagonal). Assuming $\mathbf{A}$ is invertible, we can derive the reduced-form VAR form associated with the structural 
VAR in (1):

$$
\mathbf{y}_{t}=\boldsymbol{\Phi} \mathbf{x}_{t}+\varepsilon_{t}
$$

where $\boldsymbol{\Phi}=\mathbf{A}^{-1} \mathbf{B}, \varepsilon_{t}=\mathbf{A}^{-1} \mathbf{u}_{t}$ and $\operatorname{cov}\left(\varepsilon_{t}\right)=\boldsymbol{\Omega}=\mathbf{A}^{-1} \mathbf{D} \mathbf{A}^{-1 \prime}$. Baumeister and Hamilton (2015) derive a Metropolis-Hastings algorithm that samples from A in equation (1) such that each of its columns is orthogonal to each other and satisfies the desired sign restrictions. In contrast, Canova and Nicoló (2002), Uhlig (2005) and Rubio-Ramírez et al. (2010) work with equation (2), and try to find rotations of the Cholesky factor $\mathbf{P}$ of the covariance matrix $\Omega$ that satisfy the desired sign restrictions.

While identification principles are mathematically identical when using either VAR formulation, there are major differences between the two forms on i) our ability to impose sensible prior beliefs on structural parameters, and ii) the derivation of appropriate algorithms for inference. Such issues are discussed in detail in Arias et al. (2018), Baumeister and Hamilton (2015) and Giacomini and Kitagawa (2018). The new methodology we propose in this paper results in a conceptually simple framework for set identification of VARs that allow us to set transparent (informative) priors on the structural matrix A. Most importantly, the algorithms we derive can be used in high-dimensional VARs or, more precisely, VARs of much larger dimensions considered before in the literature.

In the next Section we introduce the new methodology and associated Gibbs sampler algorithm for inference, and we outline some key components that will help speed up and stabilize (numerically) posterior sampling in high dimensions. In Section 3 we compare the new approach with various existing formulations of the VAR model, and various Bayesian algorithms for sign restrictions. In Sections 4 and 5 we undertake several important exercises using artificial and real datasets, in order to illustrate the excellent properties of the new algorithm. 


\section{VARs driven by a few, common shocks}

Our proposal stems from fundamental ideas in the factor model literature as applied to empirical macroeconomic problems: a few common forces (which in a structural setting we want to identify as "primitive shocks"; see Ramey, 2016) are driving the set of shocks to a system of $n$ endogenous variables. In order to materialize these ideas, we decompose the reduced-form VAR disturbances in equation (2) using the following static factor form

$$
\varepsilon_{t}=\Lambda \mathbf{f}_{t}+\mathbf{v}_{t}
$$

where $\boldsymbol{\Lambda}$ is an $n \times r$ matrix of factor loadings, $\mathbf{f}_{t}$ is an $r \times 1$ vector of factors, and $\mathbf{v}_{t}$ is an $n \times 1$ vector of idiosyncratic shocks. The crucial assumption here is that $n$ is large and that $r<n$ (and not necessarily $r \ll n$, as is typically assumed in the factor literature). In line with the "exact factor model" literature, we assume $\mathbf{v}_{t} \stackrel{\text { i.i.d. }}{\sim} N\left(\mathbf{0}_{n \times 1}, \boldsymbol{\Sigma}\right)$, with $\boldsymbol{\Sigma}$ a diagonal matrix. Additionally, we assume $\mathbf{f}_{t} \sim N\left(\mathbf{0}_{r \times 1}, \mathbf{I}_{r}\right)$, which means that the conditional covariance matrix of $\varepsilon_{t}$ is now of the form

$$
\operatorname{cov}\left(\varepsilon_{t} \mid \Lambda, \Sigma\right)=\Lambda \Lambda^{\prime}+\Sigma
$$

This factor model decomposition of $\Omega$ shows that, as long as $\Sigma$ is diagonal, set identification can be achieved by imposing sign restrictions on $\boldsymbol{\Lambda}$. To see this consider again a reduced-rank structural VAR representation of this model, which can be obtained by multiplying the reduced-form VAR model implied by equations (2) - (3) with the 
generalized inverse of $\boldsymbol{\Lambda}$, as follows:

$$
\begin{aligned}
\mathbf{y}_{t} & =\boldsymbol{\Phi} \mathbf{x}_{t}+\boldsymbol{\Lambda} \mathbf{f}_{t}+\mathbf{v}_{t} \\
\left(\boldsymbol{\Lambda}^{\prime} \boldsymbol{\Lambda}\right)^{-1} \boldsymbol{\Lambda}^{\prime} \mathbf{y}_{t} & =\left(\boldsymbol{\Lambda}^{\prime} \boldsymbol{\Lambda}\right)^{-1} \boldsymbol{\Lambda}^{\prime} \boldsymbol{\Phi} \mathbf{x}_{t}+\mathbf{f}_{t}+\left(\boldsymbol{\Lambda}^{\prime} \boldsymbol{\Lambda}\right)^{-1} \boldsymbol{\Lambda}^{\prime} \mathbf{v}_{t} \\
\mathbf{A}_{1} \mathbf{y}_{t} & =\mathbf{B}_{1} \mathbf{x}_{t}+\mathbf{f}_{t}+\left(\boldsymbol{\Lambda}^{\prime} \boldsymbol{\Lambda}\right)^{-1} \boldsymbol{\Lambda}^{\prime} \mathbf{v}_{t} .
\end{aligned}
$$

In the equation above, the structural VAR matrix $\mathbf{A}_{1}$ is equivalent to the generalized inverse $\left(\boldsymbol{\Lambda}^{\prime} \boldsymbol{\Lambda}\right)^{-1} \boldsymbol{\Lambda}^{\prime}$. While $\boldsymbol{\Lambda}$ is not observed, assume for a moment that we have a consistent estimator of this parameter. Given that in our exact factor model formulation the $\mathbf{v}_{t}$ are uncorrelated, the CLT in Bai (2003) suggests that for each $t$ and for $n \rightarrow \infty$ we have that $\left(\boldsymbol{\Lambda}^{\prime} \boldsymbol{\Lambda}\right)^{-1} \boldsymbol{\Lambda}^{\prime} \mathbf{v}_{t} \rightarrow 0$ making this term asymptotically negligible. Therefore, $\mathbf{f}_{t}$ in the SVAR equation (7) can be seen as a projection of the structural disturbances $\mathbf{u}_{t}$ into a lower dimensional space $\mathbb{R}^{r}$.

\subsection{A new Gibbs sampler for sign restrictions in reduced-form VARs}

Before deriving a Gibbs sampler algorithm for the high-dimensional VAR with factor structure in the residuals, we first discuss identification issues. Following Anderson and Rubin (1956) we note that $\Omega$ has $n(n+1) / 2$ free parameters while the factor decomposition in equation (4) has $n r+n$ free parameters. Therefore, the first restriction for estimation is that $n(n+1) / 2 \geq n r+n$ or that $r \leq(n-1) / 2$. This implies that - not taking into account any zero or sign restrictions on $\boldsymbol{\Lambda}$ - in a 19-variable VAR we can identify at most a reasonable number of nine factors/shocks. In practice, any zero or sign restrictions we impose on $\boldsymbol{\Lambda}$ (depending on the empirical application) will allow to lift, to a large extent, this upper bound on the number of factors. Another issue in the factor decomposition is how we identify $\boldsymbol{\Lambda}$ and $\mathbf{f}_{t}$ jointly from the likelihood. 
Lopes and West (2004) discuss this issue in detail and they follow the typical approach in identification of Bayesian factor models, which is to impose zero restrictions on the upper $r \times r$ block of $\Lambda$. Such restrictions are driven from our desire for statistical not structural - identification, and are usually suboptimal since they hardly ever fit real data precisely. ${ }^{1}$ Instead, in our case the factors $\mathbf{f}_{t}$ are equivalent to structural shocks that are uncorrelated. Therefore, we restrict their posterior to be $N\left(\mathbf{0}_{r}, \mathbf{I}_{r}\right)$. Under the assumption that $\Lambda$ will also have additional sign or zero restrictions, the structural shocks (factors) will be identified. However, even in cases where we do not have enough sign restrictions for identification of the factors, we can still fully identify the common component $\boldsymbol{\Lambda} \mathbf{f}_{t}$ as well as the decomposition of $\boldsymbol{\Omega}$ in equation (4). That is, we are always able to sample a decomposition of $\Omega$ that embeds the desired sign restrictions, even when the structural shocks are not fully identified (as long as the structural shocks are uncorrelated).

Posterior sampling in the reduced-form VAR with factor structure in the residuals is straightforward using the Gibbs sampler. This is because posterior conditional distributions have very standard form and are trivial to sample from. To see this, we now write the model using a single equation for convenience

$$
\mathbf{y}_{t}=\boldsymbol{\Phi} \mathbf{x}_{t}+\Lambda \mathbf{f}_{t}+\mathbf{v}_{t}
$$

Assume that we collect all sign and zero restrictions on $\boldsymbol{\Lambda}$ into a matrix $\mathbf{S}$, with entries +1 for positive signs, -1 for negative signs, 0 for zero restrictions, and a missing value for no restriction (we denote this case in this paper using the symbol $N A$, and in the code using the MATLAB value $N a N)$. The priors for the VAR parameters are of the

\footnotetext{
${ }^{1}$ For example, one issue with restrictions used in papers such as Lopes and West (2004) or Bernanke et al. (2005) is that the ordering of the variables in $\mathbf{y}$ plays a role on estimation of the factors. Possibly, the only exception is multi-country factor models of the form analyzed in Kose et al. (2003), where zeros in the loadings occur naturally due to the assumption of geographical grouping of country-specific economic variables.
} 
general form

$$
\begin{aligned}
\boldsymbol{\phi}_{i} \equiv \operatorname{vec}\left(\mathbf{\Phi}_{i}\right) & \sim N_{k}\left(\mathbf{0}, \underline{\mathbf{V}}_{i}\right), \\
\mathbf{f}_{t} & \sim N_{r}(\mathbf{0}, \mathbf{I}), \\
\boldsymbol{\Lambda}_{i j} & \sim \begin{cases}N\left(0, \underline{h}_{i j}\right) I\left(\Lambda_{i j}>0\right), & \text { if } S_{i j}=1, \\
N\left(0, \underline{h}_{i j}\right) I\left(\Lambda_{i j}<0\right), & \text { if } S_{i j}=-1, \\
\delta_{0}\left(\boldsymbol{\Lambda}_{i j}\right), & \text { if } S_{i j}=0, \\
N\left(0, \underline{h}_{i j}\right), & \text { otherwise, }\end{cases} \\
\sigma_{i}^{2} \sim \operatorname{inv}-\operatorname{Gamma}\left(\underline{\rho}_{i}, \underline{\kappa}_{i}\right), &
\end{aligned}
$$

for $i=1, \ldots, n, j=1, \ldots, r$, where $\boldsymbol{\Phi}_{i}$ is the $i^{\text {th }}$ row of $\boldsymbol{\Phi}, \sigma_{i}^{2}$ is the $i^{\text {th }}$ diagonal element of the matrix $\boldsymbol{\Sigma}$, and $\delta_{0}\left(\boldsymbol{\Lambda}_{i j}\right)$ is the Dirac delta function for $\boldsymbol{\Lambda}_{i j}$ at zero (i.e. a point mass function with all mass concentrated at zero). Note that our VAR specification has a diagonal covariance matrix $\boldsymbol{\Sigma}$, hence, we can follow ideas in Baumeister and Hamilton (2015) and sample the parameters of the VAR equation-by-equation. This explains why we place a prior on $\phi_{i}$, for $i=1, \ldots, n$, and not directly on the full matrix $\boldsymbol{\Phi}$.

The joint posterior of these parameters is intractable, but it is trivial to devise a Gibbs sampler that samples sequentially from conditional posteriors that are of simple form:

\section{Factor sign restrictions (FSR) algorithm}

1. Sample $\phi_{i}$ for $i=1, \ldots, n$ from

$$
\boldsymbol{\phi}_{i} \mid \boldsymbol{\Sigma}, \boldsymbol{\Lambda}, \mathbf{f}, \mathbf{y} \sim N_{k}\left(\overline{\mathbf{V}}_{i}\left(\sum_{t=1}^{T} \sigma_{i}^{-2} \mathbf{x}_{t}^{\prime} \widetilde{\mathbf{y}}_{i t}\right), \overline{\mathbf{V}}_{i}\right)
$$

where $\tilde{\mathbf{y}}_{i t}=\mathbf{y}_{i t}-\boldsymbol{\Lambda}_{i} \mathbf{f}_{t}$ and $\overline{\mathbf{V}}_{i}^{-1}=\left(\underline{\mathbf{V}}_{i}^{-1}+\sum_{t=1}^{T} \sigma_{i}^{-2} \mathbf{x}_{t}^{\prime} \mathbf{x}_{t}\right)$. 
2. Sample $\boldsymbol{\Lambda}_{i}$ for $i=1, \ldots, n$ from

$$
\boldsymbol{\Lambda}_{i} \mid \boldsymbol{\Phi}, \boldsymbol{\Sigma}, \mathbf{f}, \mathbf{y} \sim M T N_{\mathbf{a}<v e c(\boldsymbol{\Lambda})<\mathbf{b}}\left(\overline{\mathbf{H}}_{i}\left(\sum_{t=1}^{T} \sigma_{i}^{-2} \mathbf{f}_{t}^{\prime} \widehat{\mathbf{y}}_{i t}\right), \overline{\mathbf{H}}_{i}\right)
$$

where $\widehat{\mathbf{y}}_{i t} \equiv \boldsymbol{\varepsilon}_{i t}=\mathbf{y}_{i t}-\phi_{i} \mathbf{x}_{t}, \quad \overline{\mathbf{H}}_{i}^{-1}=\left(\underline{\mathbf{H}}_{i}^{-1}+\sum_{t=1}^{T} \sigma_{i}^{-2} \mathbf{f}_{t}^{\prime} \mathbf{f}_{t}\right)$, and $\underline{\mathbf{H}}_{i}=\operatorname{diag}\left(\underline{h}_{i 1}, \ldots, \underline{h}_{i r}\right)$. Here we define $\operatorname{MTN}(\bullet)$ to be the multivariate truncated Normal distribution, and $\mathbf{a}, \mathbf{b}$ are the vectors indicating the truncation points, with $i j^{\text {th }}$ element:

$$
\left(\mathbf{a}_{i j}, \mathbf{b}_{i j}\right)= \begin{cases}(-\infty, 0) & \text { if } S_{i j}=-1 \\ (0, \infty) & \text { if } S_{i j}=1 \\ (0,0) & \text { if } S_{i j}=0 \\ (-\infty, \infty) & \text { otherwise }\end{cases}
$$

for $i=1, \ldots, n, j=1, \ldots, r$.

3. Sample $\mathbf{f}_{t}$ for $t=1, \ldots, T$ from

$$
\mathbf{f}_{t} \mid \mathbf{\Lambda}, \boldsymbol{\Sigma}, \mathbf{\Phi}, \mathbf{y} \sim N\left(\overline{\mathbf{G}}\left(\boldsymbol{\Lambda} \Sigma^{-1} \widehat{\mathbf{y}}_{t}\right), \overline{\mathbf{G}}\right)
$$

where $\overline{\mathbf{G}}^{-1}=\left(\mathbf{I}_{r}+\boldsymbol{\Lambda}^{\prime} \boldsymbol{\Sigma} \boldsymbol{\Lambda}\right)$. Post-process the draws of the $T \times r$ matrix $\mathbf{f}=\left(\mathbf{f}_{1}, \ldots, \mathbf{f}_{T}\right)^{\prime}$ such that its $r$ columns (corresponding to structural shocks) are uncorrelated and standardized to unit variance. This is done by applying first the Gram-Schmidt procedure and subsequently dividing each column of $\mathbf{f}$ with its standard deviation. 
4. Sample $\sigma_{i}^{2}$ for $i=1, \ldots, n$ from

$$
\sigma_{i}^{2} \mid \mathbf{\Lambda}, \mathbf{f}, \boldsymbol{\Phi}, \mathbf{y} \sim i n v-G a m m a\left(\frac{T}{2}+\underline{\rho}_{i},\left[\underline{\kappa}_{i}^{-1}+\sum_{t=1}^{T}\left(\mathbf{y}_{i t}-\phi_{i} \mathbf{x}_{t}-\boldsymbol{\Lambda}_{i} \mathbf{f}_{t}\right)^{\prime}\left(\mathbf{y}_{i t}-\phi_{i} \mathbf{x}_{t}-\boldsymbol{\Lambda}_{i} \mathbf{f}_{t}\right)\right]^{-1}\right)
$$

A major aspect of the proposed Gibbs sampler algorithm for structural VAR inference is that all draws from the space of sign restricted reduced-rank matrices $\boldsymbol{\Lambda}$ are accepted. As explained in the next Section, this is a feature that allows us in theory to estimate VAR models with arbitrary number of endogenous variables and sign/zero restrictions. In practice, estimating very large VARs using repeated sampling (Monte Carlo) has its limitations and for that reason we next consider certain techniques that allow us to gain massively in computational efficiency.

\section{$2.2 \quad$ Priors and efficient sampling}

While the Gibbs sampler presented above is based on linear parameters and should converge smoothly and quickly, we take further steps to ensure that we come up with an overall reliable algorithm that can handle larger models with the same ease it will estimate smaller models. The main concerns come in the form of dealing with the high dimensions of $\boldsymbol{\Phi}^{2}$ and the fact that $\boldsymbol{\Lambda}$ is both latent and has to be sampled from a restricted (truncated) Normal distribution.

First, starting from $\boldsymbol{\Phi}$, sampling these parameters equation-by-equation (which is what Baumeister and Hamilton, 2015, also do) using (13), helps a lot in reducing the computational burden. Instead of having to sample one large vector of $n k$ parameters, we estimate $n$ univariate autoregressions with $k$ parameters each - a problem that is also trivial to parallelize using modern computers. We additionally follow ideas in Bhattacharya et al. (2016) and use an efficient sampler for the Normal distribution,

\footnotetext{
${ }^{2}$ For example, in a 50 variable VAR with 4 lags and an intercept this matrix has 10,000+ elements.
} 
which makes full use of the Woodbury identity in order to reparametrize the formulas in equation (13) to a more efficient form. The idea is that during sampling from the Normal distribution one has to obtain the Cholesky of a large matrix $\left(\overline{\mathbf{V}}_{i}\right.$ in our case) which requires $\mathcal{O}\left(k^{3}\right)$ algorithmic operations. The transformation of Bhattacharya et al. (2016) allows sampling with worst case algorithmic complexity of $\mathcal{O}\left(T^{2} k\right)$ operations. Therefore, gains from replacing this step with the Bhattacharya et al. (2016) approach can be very substantial as either the dimension $n$ of the VAR or the number of lags $p$ increase. In particular, as $k=n p+1$ becomes larger than $T$, a significantly smaller number of algorithmic operations will be required in order to sample from the posterior of $\boldsymbol{\Phi}$.

Second, as the dimension of $\boldsymbol{\Phi}$ increases polynomially in $n$ and $p$, computation is not the sole concern; regularization also becomes an important aspect of statistical inference. Traditionally, empirical Bayes priors such as the Minnesota prior, have been used both in reduced-form and structural VARs; see Giannone et al. (2015) and Baumeister and Hamilton (2015), respectively. However, little theory exists on the high-dimensional shrinkage properties and posterior consistency of such empirical Bayes rules. In this paper, we propose to use the horseshoe prior for sparse signals as the default choice in both small and larger VARs (Carvalho et al., 2010). The horseshoe has been shown to lead to Bayes estimates that are consistent a-posteriori, and that attain a risk equivalent to the (Bayes) oracle; see Armagan et al. (2013) and Ghosh et al. (2016) and references therein for more details. ${ }^{3}$ Using equation (9), we set the prior covariance matrix to have

\footnotetext{
${ }^{3}$ van der Pas et al. (2014) also show that the horseshoe has good frequentist properties and can attain minimax-adaptive risk up to a constant, for squared error $\left(\uparrow_{2}\right)$ loss. Recently, Kowal et al. (2019) also establish the excellent shrinkage properties of the horseshoe in a dynamic linear model for time-series data.
} 
the following hierarchical structure

$$
\begin{aligned}
\boldsymbol{\phi}_{i} \mid \sigma_{i}^{2} \tau_{i}^{2} \boldsymbol{\Psi}_{i} & \sim N_{k}\left(\mathbf{0}, \underline{\mathbf{V}}_{i}\right), \\
\underline{\mathbf{V}}_{i,(j j)} & =\sigma_{i}^{2} \tau_{i}^{2} \psi_{i, j}^{2}, \\
\psi_{i, j} & \sim \operatorname{Cauchy}^{+}(0,1), \\
\tau_{i} & \sim \operatorname{Cauchy}^{+}(0,1),
\end{aligned}
$$

where $\underline{\mathbf{V}}_{i,(j j)}$ denotes the $j^{\text {th }}$ diagonal element of prior covariance matrix $\underline{\mathbf{V}}_{i}, \mathbf{\Psi}_{i}=$ $\operatorname{diag}\left(\psi_{i, 1}^{2}, \ldots, \psi_{i, k}^{2}\right)$, and Cauchy ${ }^{+}$denotes the half-Cauchy distribution with support on the set of positive real numbers $\mathbb{R}^{+}$. The most important aspect of the horseshoe prior is that it requires absolutely no input from the researcher, while retaining at the same time its excellent shrinkage properties. There are various reparametrizations of this prior and associated MCMC sampling schemes. Here we follow Neal (2003) and use a slice sampler (within our main Gibbs sampler algorithm) that allows to update $\psi_{i,(j j)}$ and $\tau_{i}$ efficiently in high-dimensions.

Third, a major challenge in the proposed algorithm is that in Step 2, $\boldsymbol{\Lambda}_{i j}$ has to be sampled from a multivariate truncated Normal distribution which is notoriously hard to simulate from (Geweke, 1996). In order to deal with this significant computational challenge, we follow Geweke (1996) and sample each $\boldsymbol{\Lambda}_{i j}$ conditional on all remaining $\boldsymbol{\Lambda}_{-i j}$ elements. Most importantly, we adopt an exact minimax tilting method for generating i.i.d. data from a truncated normal distribution that was recently proposed by Botev (2017). This method allows orders of magnitude computational improvements relative to the truncated normal sampler proposed by Geweke (1996). ${ }^{5}$

\footnotetext{
${ }^{4}$ Note that the prior covariance matrix of $\phi_{i}$ is a function of the VAR variance $\sigma_{i}^{2}$. This is done in order to enhance numerical stability when the endogenous variables in the VAR are measured in different units (scaling issue), even though in practice one could easily specify the prior to be independent of $\sigma_{i}^{2}$ (which would be essential in case we want to specify $\sigma_{i}^{2}$ to be time-varying, using for instance a stochastic volatility specification.

${ }^{5}$ Further gains in efficiency are possible by adopting the interweaving strategies for factor models
} 
Given absence of prior information on the variance of $\boldsymbol{\Lambda}$ (above and beyond the information we have on the sign restrictions) we set $\underline{h}_{i j}=4$ in the remainder of the paper, which is a noninformative choice for the parameters of a loadings matrix.

All the considerations above ensure that the algorithm proposed in the previous subsection is not only fast, but is also numerically stable and can scale up to much larger VAR dimensions than ever considered before in the literature. Note that there are remaining prior settings and algorithmic steps (those involving parameters $\mathbf{f}_{t}$ and $\boldsymbol{\Sigma}$ ), but these steps are already computationally trivial and may not be made more efficient. Additionally, the prior for $\mathbf{f}_{t}$ is fixed by the required identification restrictions, and the $\boldsymbol{\Sigma}$ are integrated out with fairly noninformative values (we set $\rho_{i}=1$ and $\kappa_{i}=0.1$ for all $i$, in all VARs estimated in this paper). Exact details of the proposed algorithm, including the additional enhancements described in this subsection, are provided in the online Appendix.

\subsection{Likelihood-based testing of identifying assumptions in VARs}

Our modeling assumption that the structural shocks $\mathbf{f}_{t}$ are latent parameters that have to be estimated from the likelihood, has an enormous implication: sign restrictions become part of the model likelihood and can be explicitly tested the same way economists test other parametric restrictions in regression models (e.g. inequality constraints as in Geweke, 1996). This concept might not make sense for economic shocks that are indisputable such as the effects of an aggregate demand/supply shock, however, there are cases of shocks where the expected sign might not be known a-priori with certainty. Alternatively, a researcher might want to statistically test the plausibility of certain zero restrictions, or simply compare the performance of two different VAR models (e.g. with proposed in Kastner et al. (2017), although we do not adopt such a strategy in this paper. 
different number of lags and/or variables) given the same set of identifying restrictions. In our setting, all these cases can be tested explicitly using marginal likelihoods or Bayes factors. Parametric (i.e. zero or sign) restrictions on $\boldsymbol{\Lambda}$ that agree with the information in the data will result in higher marginal data likelihood relative to restrictions that are not supported by the data. Put differently, given the decomposition in equation (4), plausible restrictions in $\boldsymbol{\Lambda}$ will result in estimation of a more precise unconditional VAR covariance matrix $\Omega$.

Marginal likelihoods are not numerically stable in high-dimensional VARs (Giannone et al., 2015) and they can be demanding to compute even in smaller VARs with many layers of latent parameters (e.g. hierarchical priors like the horseshoe; stochastic variances; Markov-switching coefficients). In order to deal with this computational aspect, we propose to calculate instead the Deviance Information Criterion (DIC) of Spiegelhalter et al. (2002) as a default criterion for comparing parametric restrictions on $\boldsymbol{\Lambda}$. For the matrix of VAR model parameters $\boldsymbol{\Theta}=\left(\boldsymbol{\Phi}, \boldsymbol{\Lambda},\left\{\mathbf{f}_{t}\right\}_{t=1}^{T}, \boldsymbol{\Lambda}\right)$ the DIC is defined as the quantity

$$
D I C=-4 E_{p(\mathbf{\Theta} \mid \mathbf{y})}(\log f(\mathbf{y} \mid \mathbf{\Theta}))+2 \log f(\mathbf{y} \mid \widehat{\mathbf{\Theta}})
$$

where $\log f(\mathbf{y} \mid \boldsymbol{\Theta})$ is the $\log$ of the likelihood function implied by the regression in (8). The first term in the criterion above is the expectation of the likelihood w.r.t the parameter posterior, and can be obtained numerically using Monte Carlo integration by simply evaluating the likelihood at each MCMC draw from the posterior of the parameters $\boldsymbol{\Theta}$. The second term is the likelihood function evaluated at an estimate $\widehat{\boldsymbol{\Theta}}$ of high posterior density (typically posterior mean or mode). As with all other information criteria used in statistics/econometrics, lower values signify better fit. The DIC is not a first-order approximation to the marginal likelihood, in the same way that the Bayesian Information Criterion (BIC) is. The marginal likelihood, also known as 
prior predictive distribution, addresses the issue of how well the data are predicted by the priors. In this sense, the DIC is a criterion that is closely related to measuring fit according to the posterior predictive distribution, rather than marginal likelihoods. As a result, for the purpose of assessing the fit of a VAR that is intended to be used for out-of-sample projections (impulse responses, forecast error variance decompositions etc), the DIC can be considered as a more appropriate predictive measure of fit compared to marginal likelihoods or alternative in-sample measures of fit.

\section{Interpretation of the new Gibbs sampler for sign restrictions}

The proposed methodology builds on several previous attempts and efforts for structural VAR identification. ${ }^{6}$ Gorodnichenko (2005) specified an identical VAR model with reduced-rank decomposition of the disturbance terms. The main idea behind proposing this specification was to replace standard block diagonal restrictions in VARs (Bernanke and Blinder, 1992), with a more parsimonious identification scheme that imposes less (possibly unreasonable) zero restrictions. More recently, Matthes and Schwartzman (2019) also specify a closely related VAR model in order to identify the structural impact of sectoral dynamics on GDP. Their identification is via a factor structure on the residuals that has the additional assumption of allowing for correlation within industries but no correlation across industries.

Similarly, Stock and Watson (2005a) specify a more general factor-augmented VAR (FAVAR) and discuss in detail how various identification schemes fit in this setting. They also note (Stock and Watson, 2005a, Section 3.5) that the sign restrictions identification

\footnotetext{
${ }^{6}$ To be exact, the VAR with factor structure is inspired by the panel data literature, and such structure has been used before in multi-country VARs; see Stock and Watson (2005b).
} 
scheme proposed by Uhlig (2005) also fits the FAVAR framework. ${ }^{7}$ In practice, the FAVAR not only imposes a reduced-rank decomposition of the errors using a matrix $\boldsymbol{\Lambda}$ but also assumes that VAR coefficients $\boldsymbol{\Phi}$ have a similar factor structure using the same projection matrix $\boldsymbol{\Lambda}$. This means that the structural moving average form of the FAVAR model is a convolution of several matrices (Stock and Watson, 2005a, eq. (22)) and imposing sign restrictions on the covariance matrix will also restrict the signs of the VAR lags in a complicated nonlinear way. Imposing sign restrictions on VAR coefficients is not advisable as, in general situations, such restrictions won't be supported by evidence in the data. In contrast, our simpler formulation does not impose sign or other subjective restrictions on the matrix of VAR lags $\boldsymbol{\Phi} .^{8}$

Our current factor-VAR formulation and algorithm are connected to existing Bayesian MCMC algorithms for inference in reduced-form VAR, structural VAR (SVAR), and structural vector moving average (SVMA) models. We next explain such connections to some key, recently proposed algorithms.

\subsection{Connection to existing MCMC samplers for reduced-form VARs}

The vast majority of papers working with sign restrictions build on the reduced-form VAR in equation (2) under the assumption that $\varepsilon_{t} \sim N(\mathbf{0}, \boldsymbol{\Omega})$. The main idea behind such algorithms is that, in a first step, it is trivial in a reduced-form VAR to simulate from the joint posterior of the parameters $(\boldsymbol{\Phi}, \boldsymbol{\Omega})$. Then, at a second step, for each sample of $\boldsymbol{\Omega}$ its unique Cholesky factor $\mathbf{P}$ is obtained, and rotated using an $n \times n$ orthogonal matrix Q. The final rotated matrix $\boldsymbol{\Xi}=\mathbf{P Q}$ might, or might not, satisfy the sign restrictions we want to impose. Canova and Nicoló (2002), Uhlig (2005) and Rubio-Ramírez et al.

\footnotetext{
${ }^{7}$ For an application of this idea, see Ahmadi and Uhlig (2015).

${ }^{8}$ Instead, our algorithm only assumes that regularization restrictions in this matrix occur in a datarigorous way by means of the hierarchical horseshoe prior.
} 
(2010) have all proposed algorithms for generating orthogonal matrices Q by drawing uniformly from the space of $n \times n$ orthogonal matrices, with the latter paper proposing the most computationally efficient scheme.

Nevertheless, the step of these algorithms that requires to verify whether $\boldsymbol{\Xi}$ satisfies the required restrictions belongs to the general class of accept/reject algorithms, which are notoriously inefficient. In high dimensions (e.g. $n>7$ ) it becomes increasingly difficult to find random rotations of $\mathbf{P}$ that satisfy the desired sign restrictions. Similarly, once we incorporate sign restrictions in more empirically relevant VARs that feature stochastic variation of the covariance matrix (e.g. using Markov switches as in Sims and Zha, 2006), such accept/reject algorithms have to be applied every period there is a regime change, increasing substantially the computational burden. Finally, existing algorithms identify sets of models that comply with the sign restrictions, which means that they do not allow to test explicitly whether the likelihood specification (e.g. number of lags) or selected priors, support such restrictions.

In contrast, our proposed algorithm fully exploits the notion that economic variables co-move, in order to build on a parsimonious VAR formulation where parameters and sign restrictions are sampled together in one step. Apart from the obvious fact that in our Gibbs sampler all sign restrictions are accepted, there is the benefit that the sign restrictions become part of the VAR likelihood. This means that we can use the suggested deviance information criterion to explicitly test whether different sets of sign restrictions are actually supported by the data. All these points become more clear later, in the Monte Carlo and empirical sections of this paper. Finally, as Adalid et al. (2019) demonstrate, this algorithm is appropriate for high-dimensional reduced-form VARs featuring time-varying parameters and stochastic volatilities, even when using a combination of zero and sign restrictions. 


\subsection{Connections to existing MCMC samplers for structural VARs}

Many economists argue that it is desirable to work with the structural VAR in equation (1). For that reason, general Bayesian algorithms for inference have been proposed; see Waggoner and Zha (2003) and Canova and Pérez Forero (2015). When it comes specifically to identification using sign restrictions, Baumeister and Hamilton (2015) proposed a Metropolis-Hastings algorithm that samples the contemporaneous structural matrix A along with the remaining SVAR coefficients $\mathbf{B}$ and $\mathbf{D}$. The way they do this is by specifying natural conjugate priors for $\mathbf{B}, \mathbf{D}$ in order to integrate analytically their effect from the joint posterior of all SVAR parameters $p(\mathbf{A}, \mathbf{B}, \mathbf{D} \mid \mathbf{y})$. Their sampler then involves sampling only $p(\mathbf{A} \mid \mathbf{y})$ using Metropolis-Hastings, and then $p(\mathbf{B} \mid \mathbf{D}, \mathbf{y})$ and $p(\mathbf{D} \mid \mathbf{y})$ using Monte Carlo integration. In that respect, their algorithm shares a fundamental similarity with our algorithm: the sign restrictions are sampled along with the other model parameters, and the "fit" of the sign restrictions in the data will affect those other parameter estimates. The main advantage of estimating the SVAR instead of the reduced-form VAR is that priors can be elicit in an explicit way directly on the structural parameters.

Despite these benefits, the Baumeister and Hamilton (2015) approach has the restriction of relying exclusively on the use of a natural conjugate prior, which does not allow the estimation of interesting cases such as heteroskedastic VARs, or VARs with Markov switching dynamics in the intercept and autoregressive coefficients, or the specification of full Bayes priors that make more sense as the dimension of the VAR gets larger. In any case, we argue here that it would be trivial to combine the approach in Baumeister and Hamilton (2015) with the reduced-rank SVAR implied by our model:

$$
\mathbf{A}_{1} \mathbf{y}_{t} \approx \mathbf{B}_{1} \mathbf{x}_{t}+\mathbf{f}_{t}
$$


in order to obtain a more efficient algorithm for high-dimensional inference in SVARs. Even in the high-dimensional case with large $n$, all is required from the Metropolis-Hasting step of their algorithm is to sample an $n \times r$ matrix, which should be computationally feasible for small $r$.

\subsection{Connections to existing MCMC samplers for structural VMAs}

Not many economists have historically attempted to estimate VMA models, due to their inherent demands in estimation: all endogenous variables are a function of latent parameters and (lags of) latent shocks. A recent exception, with focus on identification via sign restrictions, is the study of Plagborg-Møller (2019) who relies on a certain reparametrization combined with a Hamiltonian Monte Carlo sampler. The benefits of this approach is that one can directly put priors on the impulse response functions, since the parameters of the VMA form measure in an explicit way the effect of each structural shock on the observables. Unfortunately, the proposed HMC sampler needs to be expertly tuned to the specific data and problem at hand, which means that it is far from a generic algorithm that could be used with any data (small or larger). In any case, the VAR we propose in this paper ${ }^{9}$ admits the following $\operatorname{VMA}(q)$ representation

$$
\begin{aligned}
\mathbf{y}_{t} & =\boldsymbol{\mu}+\sum_{j=0}^{q} \boldsymbol{\Psi}_{j} \boldsymbol{\Lambda} \mathbf{f}_{t-j}+\sum_{j=0}^{q} \boldsymbol{\Psi}_{j} \mathbf{v}_{t-j} \\
& \approx \boldsymbol{\mu}+\sum_{j=0}^{q} \boldsymbol{\Psi}_{j} \boldsymbol{\Lambda} \mathbf{f}_{t-j}
\end{aligned}
$$

\footnotetext{
${ }^{9}$ For simplicity we rewrite our model in $\operatorname{VAR}(1)$ form
}

$$
\mathbf{y}_{t}=\boldsymbol{\Phi}_{0}+\boldsymbol{\Phi}_{1} \mathbf{y}_{t-1}+\mathbf{\Lambda} \mathbf{f}_{t}+\mathbf{v}_{t}
$$

where $\boldsymbol{\Phi}_{0}$ is the $n \times 1$ vector of intercepts, and $\boldsymbol{\Phi}_{1}$ is the $n \times n$ matrix of coefficients on first lagged variables. It is then trivial to derive the $\operatorname{VMA}(\infty)$ form implied by the $\operatorname{VAR}(1)$ and truncate it to $q$ lags. In order to derive the VMA associated with the general case of the $\operatorname{VAR}(p)$ model with $p>1$, we can use the standard result that every $\operatorname{VAR}(p)$ model can be written in $\operatorname{VAR}(1)$ companion form. 
where $\boldsymbol{\mu}=\left(\mathbf{I}-\boldsymbol{\Phi}_{1}\right)^{-1} \boldsymbol{\Phi}_{0}$ and $\boldsymbol{\Psi}_{j}=\boldsymbol{\Phi}_{1}^{j}$. As long as the are enough sign restrictions on $\boldsymbol{\Lambda}$ and we normalize $\mathbf{f}_{t}$ to have identity covariance (as we already do in the VAR case), we should be able to directly specify the reduced-rank VMA model in equation (26) and use the algorithm of Plagborg-Møller (2019) to estimate the model in high dimensions.

\section{Simulation study}

In this section we examine closely the properties of the new algorithm using artificial data. Our experiment involves generating multivariate time series from a data generating process (DGP) that fully matches equation (8), and estimating parameters and impulse response functions based on these generated series. We first implement this experiment assuming that we estimate a correctly specified model. We then consider various cases of misspecification errors during the estimation process - that is, we estimate models that do not perfectly match the correct DGP. The DGP is of the form

$$
\begin{aligned}
\mathbf{y}_{t} & =\widehat{\boldsymbol{\Phi}} \mathbf{x}_{t}+\widehat{\Lambda} \mathbf{f}_{t}+\mathbf{v}_{t}, \text { for } t=1, \ldots, \widehat{T}, \\
\mathbf{v}_{t} & \sim N(\mathbf{0}, \widehat{\Sigma}), \quad \mathbf{f}_{t} \sim N(\mathbf{0}, \mathbf{I}), \\
\mathbf{y}_{(-p+1): 0} & =\mathbf{0}, \quad p=12, \quad r=3 .
\end{aligned}
$$

The DGP parameters $\widehat{\boldsymbol{\Phi}}, \widehat{\boldsymbol{\Lambda}}, \widehat{\boldsymbol{\Sigma}}$ are based on estimates of a VAR on real data. We first collect monthly data on 14 monthly macroeconomic variables ${ }^{10}$ for the US over the period 1965M1 - 2007M12, providing $\widehat{T}=516$ observations, before transforming variables to stationarity or taking lags. ${ }^{11}$ We next obtain $\widehat{\boldsymbol{\Phi}}$ as the OLS estimate on an unrestricted $\operatorname{VAR}(12)$ estimated with these 14 variables. We next estimate the first

\footnotetext{
${ }^{10}$ The variables are: 1) real GDP, 2) GDP deflator, 3) federal funds rate, 4) commodity price index, 5) total reserves, 6) nonborrowed reserves, 7) S\&P 500, 8) M1 ,9) unemployment rate , 10) industrial production , 11) employment , 12) CPI , 13) core CPI , 14) core PCE. More details on these variables is provided in the online Appendix.

${ }^{11}$ In practice, we generate $\widehat{T}+1000$ observations and we discard the first 1000 observations.
} 
$r=3$ principal components of the OLS residuals, and we obtain $\widehat{\Lambda}$ as the OLS estimate in a regression between the VAR residuals and their principal components. Finally, the residuals from this latter regression provide the elements of the diagonal matrix $\widehat{\Sigma}$, by means of equation-by-equation application of the usual least squares formula for the variance.

While it is not possible, or even interesting, to print all estimates $\widehat{\boldsymbol{\Phi}}$ used as input in the DGP, it is instead interesting to look at the estimates $\widehat{\Lambda}$ of $\boldsymbol{\Lambda}$ based on the procedure described above. This is because both the signs and the magnitudes of the implied IRFs in the true DGP will be affected by those estimates. Panel (A) of Table 1 shows the OLS estimates, where the diagonal is normalized to be one, by dividing each element in the $m^{\text {th }}$ column of $\widehat{\Lambda}$ with the original value of its $m^{t h}$ element, $m=1,2,3$. While this matrix is the outcome of using real data and applying simple principal components plus OLS estimation (which carry no economic restrictions), the signs implied by it allow us to classify the three pseudo-shocks as aggregate supply, aggregate demand, and monetary policy, respectively. The estimated magnitudes of course are not necessarily economically meaningful. For example, if we do believe that we can label the third estimated shock as a monetary policy shock, then the entries in Table 1 suggest that a one per cent increase in the federal funds rate will total reserves to decrease by 3.48 per cent. Nevertheless, this is an exercise where our main aim is to check the numerical precision of the new algorithm, so the estimates in panel (A) of Table 1 are perfectly valid inputs for a DGP. Finally, Panel (B) of Table 1 shows the imposed sign restrictions. These comply with the signs imposed in the DGP, and in 11 instances we do not impose any sign restrictions (these entries are denoted as $N A$ ). 


\begin{tabular}{lcccccc}
\hline & (A) TRUE PARAmETER VAluts & \multicolumn{2}{c}{ (B) SigN RESTRICTIONS } \\
Variable & $1^{\text {st }}$ shock & $2^{\text {nd }}$ shock & $3^{\text {rd }}$ shock & $1^{\text {st }}$ shock & $2^{\text {nd }}$ shock & $3^{\text {rd }}$ shock \\
\hline \hline real GDP growth & 1.00 & -1.39 & -0.87 & + & - & - \\
GDP deflator inflation & 1.42 & 1.00 & -0.71 & + & + & - \\
Fed funds rate & 0.49 & -0.28 & 1.00 & $\mathrm{NA}$ & $\mathrm{NA}$ & + \\
Commodity prices & 0.16 & 0.16 & -0.45 & $\mathrm{NA}$ & $\mathrm{NA}$ & - \\
Total reserves & -0.61 & 0.22 & -3.48 & $\mathrm{NA}$ & $\mathrm{NA}$ & $\mathrm{NA}$ \\
Nonborrowed reserves & -0.91 & 0.25 & -3.37 & $\mathrm{NA}$ & $\mathrm{NA}$ & - \\
Stock prices & -0.25 & -0.30 & -0.82 & $\mathrm{NA}$ & $\mathrm{NA}$ & - \\
M1 & -1.03 & -0.48 & -1.27 & - & - & - \\
Unemployment & -0.63 & 0.51 & 0.43 & - & + & + \\
Industrial production & 1.12 & -1.34 & -0.87 & + & - & - \\
Employment & 0.88 & -1.00 & -1.01 & + & + & - \\
CPI inflation (total) & 1.44 & 1.01 & -0.75 & + & + \\
CPI inflation (core) & 1.05 & 0.49 & -1.12 & + & + \\
PCE inflation (core) & 1.05 & 0.57 & -0.80 & + & + \\
\hline
\end{tabular}

Notes: Panel (A) shows true parameter values used as input in the data generating process (DGP), while panel (B) shows the sign restrictions imposed during econometric estimation using each artificial dataset from the DGP. Entries in panel (B) show the restrictions imposed: + for positive sign; - for negative sign; NA for no restriction.

Table 1: OLS estimates $\widehat{\Lambda}$ used in the DGP, and sign restrictions used for estimation

For estimation purposes we assume five different scenarios: one correctly specified case and four misspecified cases. These are denoted as C1-C5, and are defined as follows:

C1 Correctly specified model with $n=14$ dependent variables, $p=12$ lags, $r=3$ shocks.

C2 Misspecified model with $n=8$, using the first eight variables in Table B1 in the online Appendix. All other settings are correct, that is, $p=12$ lags, $r=3$ shocks.

C3 Misspecified model with $p=2$ lags. All other settings are correct, that is, $n=14$ 
and $r=3$.

C4 Misspecified model with $r=2$ shocks, using only the restrictions on the first two shocks in panel (B) of Table 1. All other settings are correct, that is, $n=14$ and $p=12$.

C5 Misspecified model with $r=4$ shocks, using an additional shock. ${ }^{12}$ All other settings are correct, that is, $n=14$ and $p=12$.

We generate 500 datasets of size $T=516$ and we obtain posterior mean estimates of all parameters, IRFs and DICs from all five cases above. Results presented next are based on the distribution of the posterior means over these 500 artificial datasets.

Before analyzing the quantitative results, it is important to stress again that our algorithm allows not only to test for model fit with respect to lag length, but also explicitly test the plausibility of the number of identified shocks and associated sign restrictions imposed. Table 2 shows the value of the deviance information criterion attained by each of the five cases. Because case $\mathrm{C} 2$ refers to a VAR with $n=8$, it is impossible to directly compare it with the other four cases that assume $n=14 .{ }^{13}$ For that reason we present two DIC metrics, the full one based on all $n=14$ variables (with no value for $\mathrm{C} 2$ ) and a reduced DIC which is the same formula evaluated only on the first eight VAR equations (which are common to all five cases). These are labeled in Table 2 as $D I C_{14}$ and $D I C_{8}$, respectively. According to both subsets of criteria we find that the correctly specified estimated model is the best one as it attains the lowest DIC value. Interestingly, the case where we estimate an additional fourth shock (C5), doesn't seem to harm estimation accuracy; at least not as much as the case of estimating one less shock (C4). By far the worst type of misspecification seems to be the one related

\footnotetext{
${ }^{12}$ This fourth shock is identified using the randomly selected vector of restrictions $s=$ $[+,+,+,-,+, N A, N A,-,+,+,+,+,+,+]$.

${ }^{13}$ All information criteria can only be used to compare models with the same dependent variable $\mathbf{y}$.
} 
to the lag-length. This is a characteristic of the VAR model rather than a "problem" with the specific algorithm or prior. As long as the true DGP has $p=12$ important lags, estimating the VAR with $p=2$ provides a huge loss of information. In contrast, reducing the VAR from $n=14$ (which is the truth in the DGP) to $n=8$ as in case $\mathrm{C} 2$, harms much less the fit of our VAR. This last evidence is not enough on its own to suggest that getting the lags correctly specified is more important than deciding on the correct number of endogenous variables. A large-scale comparison where we also restrict $\mathbf{y}_{t}$ to be much smaller (e.g. $n=3$ ) would be illuminating, but such numerical exercise is beyond the scope of this paper.

\begin{tabular}{cccccc}
\hline & $\mathrm{C} 1$ & $\mathrm{C} 2$ & $\mathrm{C} 3$ & $\mathrm{C} 4$ & $\mathrm{C} 5$ \\
\hline \hline$D I C_{14}$ value & 7393.14 & $\mathrm{n} / \mathrm{a}$ & 27241.13 & 11859.45 & 9037.44 \\
$D I C_{8}$ value & 15300.63 & 17094.28 & 28630.21 & 27981.48 & 19269.55 \\
\hline
\end{tabular}

Notes: $D I C_{14}$ is the deviance information criterion applied jointly to all 14 VAR equations. $D I C_{8}$ is the same criterion applied jointly only to the first 8 VAR equations. Case $\mathrm{C} 2$ does not have a $D I C_{14}$ value because it assumes that the VAR has $n=8$ variables.

Table 2: DIC values attained by the correctly specified and misspecified models estimated on artificial data

Next, we want to find out how precise the empirical estimates are relative to the values of the VAR parameters that we fed into the DGP. Since our focus is on impulse response analysis, we compare precision of the estimated impulse response functions. IRFs are combinations of all VAR parameters $\boldsymbol{\Phi}, \boldsymbol{\Lambda}, \mathbf{f}, \boldsymbol{\Sigma}$, therefore comparing their precision provides a convenient summary of overall estimation precision in our VAR model. Figure 1 shows the responses of the first three variables in the VAR to the three identified pseudo-shocks, in the correctly specified case (C1). Green solid lines 
are medians over the posterior IRFs in the 500 estimated VARs using an equal number of artificial datasets. Shaded areas show the $90 \%$ probability bands of these IRFs. Finally, black dashed lines show the true IRFs implied by the parameters we have fed in the DGP. The $90 \%$ bands always include the true IRF, which suggests that estimation precision is satisfactory. The online Appendix shows identical graphs for the four misspecified cases C2-C5. These graphs become a visual confirmation of the numerical results in Table 2, that is, case C5 quite precisely captures the path of the true IRFs, while case C3 results in the larger estimation errors.
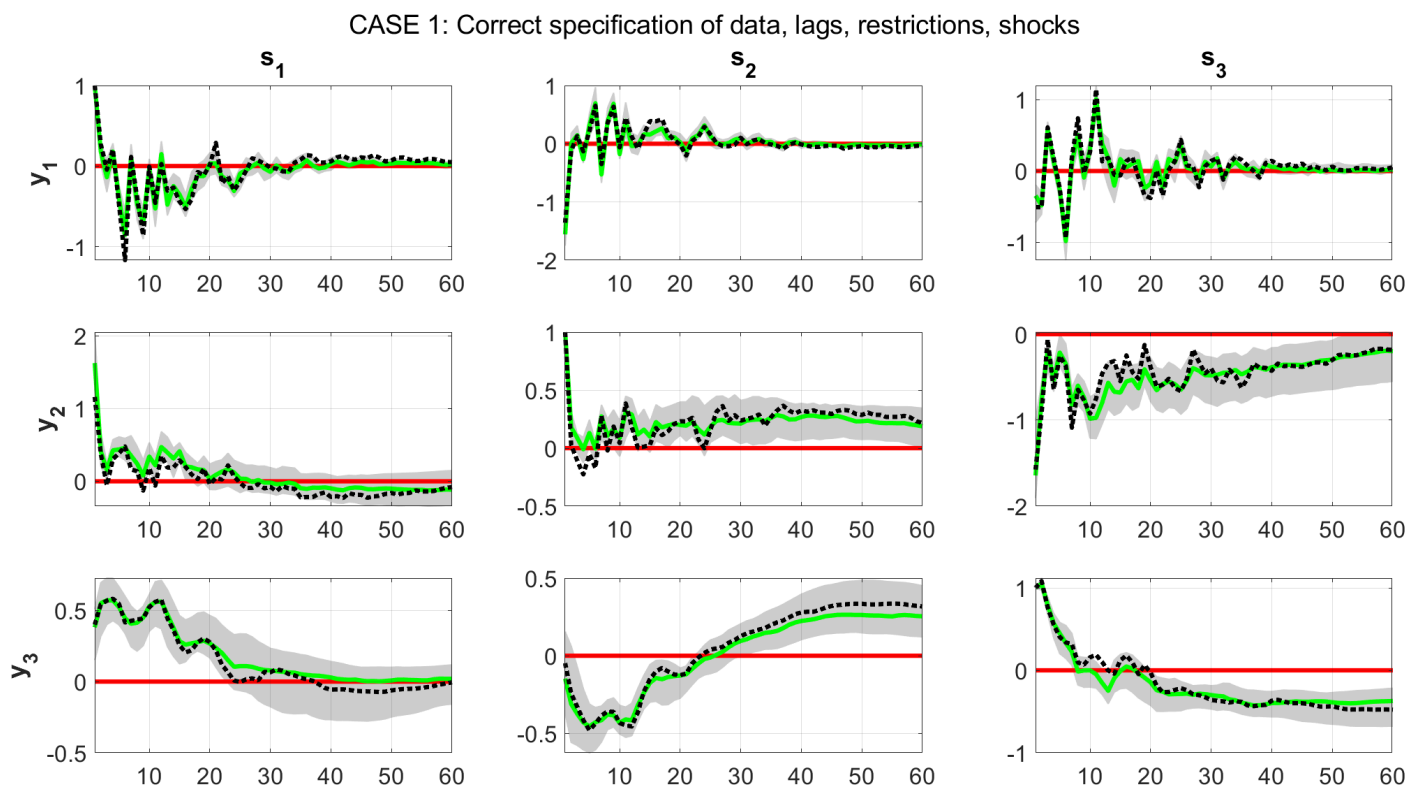

Figure 1: Impulse response functions of the first three artificially generated variables (denoted as $\left.y_{1}, y_{2}, y_{3}\right)$ in response to the three identified shocks (denoted as $s_{1}, s_{2}, s_{3}$ ) in model C1 (correctly specified model). The green solid lines show the posterior median IRFs over the 500 Monte Carlo iterations, and the gray shaded areas their associated 90\% bands. The true IRFs based on the DGP are shown using the black dashed lines. 


\subsection{How fast is the new algorithm?}

The next Section makes clear that in the context of the empirical application in Furlanetto et al. (forthcoming), the new algorithm is multiple times faster than the algorithm of Rubio-Ramírez et al. (2010) in a six-variable VAR with five identified shocks. Nevertheless, it would be interesting to use artificial data in order to provide more thorough evidence on how fast the factor sign restrictions algorithm is, and how large a VAR it can scale to. For that reason we generate artificial data from the same DGP described in equations (27)-(28) for various values of the key parameters that affect the dimensionality of the VAR, namely $T, n$ and $r$. Given that we go to very large values of $n$, we fix $p=1$, despite the fact that higher values of this parameter also increase the size of the VAR (and computation times) substantially. We do this in order to ensure that we can always generate stationary VARs easily. For the purposes of this exercise we set $\boldsymbol{\Phi}=0.9 \mathbf{I}_{n}$ (i.e. a stationary VAR for $\left.p=1\right), \boldsymbol{\Lambda}_{i j} \sim U(-1,1)$ and $\Sigma_{i} \sim U(0,1)$, for all $i=1, \ldots, n$ and $j=1, \ldots, r$. During estimation we impose $n k$ sign restrictions, simply by obtaining the signs of the randomly generated matrix $\Lambda .{ }^{14}$

\footnotetext{
${ }^{14}$ The purpose of this exercise is not to estimate meaningful restrictions, rather just to measure times. In this case, we impose the maximum number of restrictions possible on $\boldsymbol{\Lambda}$ in order to test our algorithm in a worst-case scenario where all $n k$ of its elements have to be generated from the truncated Normal posterior distributions.
} 


\begin{tabular}{ccccccc}
\hline & & $T=200$ & & & & \\
& $n=15$ & $n=50$ & $n=100$ & $n=15$ & $n=50$ & $n=100$ \\
\hline \hline$r=3$ & 1 & 2 & 6 & 4 & 11 & 23 \\
$r=10$ & 1 & 4 & 8 & 4 & 12 & 23 \\
$r=20$ & $\mathrm{NA}$ & 6 & 11 & NA & 15 & 25 \\
\hline
\end{tabular}

Table 3: Computer time in minutes (defined as (seconds/60), rounded to the nearest integer) for obtaining 10,000 post-burn-in draws (12,000 in total) using various VAR sizes. Here $T$ is the number of observations, $n$ the number of endogenous variables, and $r$ the number of shocks. All VARs have $p=1$ lag.

Table 3 shows the average, over 10 Monte Carlo iterations, machine time in minutes (defined as the total estimation time in seconds divided by 60 and then rounded to the nearest integer) needed to obtain 10,000 draws from the posterior of all parameters after discarding 2,000 draws (hence, 12,000 draws in total). These results show that in a huge-dimensional VAR with $n=100$ series, $T=500$ observations, and $r=20$ shocks, it only takes 25 minutes to obtain 10,000 draws from all parameter matrices, including the 1000 sign-restricted elements in $\Lambda$. For the smaller model with $n=15$ - which is already much larger than the vast majority of models considered in the sign restrictions literature - it only takes less than five minutes to obtain the same number of draws when $T=500$, and only one minute when $T=200$. These fantastic timings justify our choice to focus on carefully developing a Gibbs sampler that is computationally efficient. ${ }^{15}$

The results above are based on code written in MATLAB2019b and run in a personal

\footnotetext{
${ }^{15}$ The Gibbs sampler typically loses efficiency when there is high correlation in the samples from the posterior. In the Appendix we show that, in order to draw $\boldsymbol{\Lambda}_{i j}$ from univariate (instead of the intractable multivariate) truncated Normal conditionals, we need to condition on $\boldsymbol{\Lambda}_{-i j}$, i.e. the set of all elements of $\boldsymbol{\Lambda}$ excluding the $i j^{t h}$. This conditioning increases correlation relative to sampling directly the full matrix $\boldsymbol{\Lambda}$. However, inefficiency factors for the Gibbs sampler in our linear factor-VAR specification are still quite low (MCMC diagnostic results are available upon request). Additionally, given our ability to obtain quickly tens of thousands of draws from the posterior, concerns about possible correlation of draws can be alleviated by doing "thinning" - i.e. the procedure of storing only every $10^{t h}$ or so sample from the posterior, depending on the severity of the (auto)correlation.
} 
computer with Intel Core i7 $8700 \mathrm{~K}$, tuned at $4.9 \mathrm{Ghz}$, and $32 \mathrm{~GB}$ of RAM. Note that the Gibbs sampler algorithm iterates over each VAR equation independently and, thus, we can achieve significant speed improvements by taking advantage of parallel processing abilities of modern computers and high-performance clusters (HPCs). In MATLAB this is as simple as replacing for loops with parfor loops. Therefore, the algorithm indeed allows the estimation of arbitrarily large VAR models, as it is claimed in the Introduction.

In practical situations, the only issue that might inhibit the performance of the algorithm (and any Monte Carlo-based algorithm, to that effect) is the fact that in very large dimensions we may be sampling parameters $\boldsymbol{\Phi}$ in a region of the posterior that implies nonstationarity of the VAR. In order to make sense out of impulse response functions, forecast error variance decompositions, historical decompositions etc, we need to make sure we maintain only samples from the posterior which are stationary. For that reason we need to stress that, throughout our experiments, the horseshoe prior does a great job (especially relative to a subjectively chosen Minnesota prior) in shrinking the coefficients $\boldsymbol{\Phi}$ towards a more numerically stable region of their posterior, where the VAR model is stationary. We could potentially thoroughly examine this claim about the properties of the horseshoe prior, using additional Monte Carlo experiments. However, such an exercise is beyond the scope of this paper (which is focused on inference on structural parameters and not autoregressive coefficients) and the reader is referred for further evidence to the key theoretical and empirical papers around this prior cited in subsection 2.2 . 


\section{$5 \quad$ Empirics}

\section{$5.1 \quad$ Measuring optimism shocks}

Before we use the new algorithm for building a high-dimensional structural VAR model for the US identified with sign restrictions, we undertake an empirical exercise that will shed first light on the performance of the new algorithm on real data. This empirical exercise is based on Section 6 of Arias et al. (2018). These authors use the example in Beaudry et al. (2011) in order to compare their novel importance sampling algorithm to the penalty function approach (PFA) of Mountford and Uhlig (2009). For the sake of comparability, we maintain their empirical setting, and for that reason we estimate VAR(4) models using the following five dependent variables: adjusted TFP, stock prices, consumption, the real interest rate, and hours worked. We identify a single optimism shock by restricting contemporaneously TFP to have a zero response, and stock prices to react positively. The signs in the remaining three variables are not restricted. Panels (a) and (b) in Figure 2 show the estimated responses using the PFA and importance sampling algorithms, respectively. These two panels are identical to panels (a) and (b) in Figure 1 of Arias et al. (2018). The main point these authors make in their study is that the PFA algorithm ends up distorting the responses of stock prices, consumption and hours. Once their proposed importance sampling algorithm is considered, the significant responses found in Beaudry et al. (2011) disappear. 


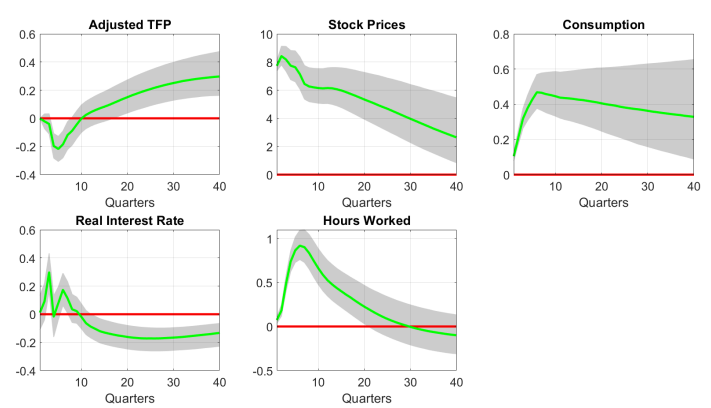

(a) Mountford and Uhlig (2009) algorithm

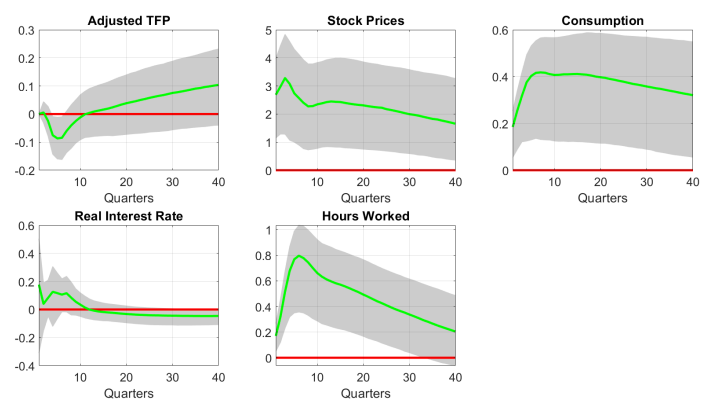

(c) Factor sign restrictions algorithm
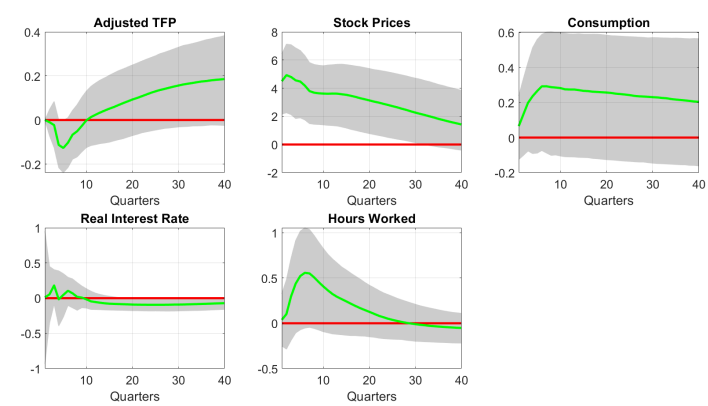

(b) Arias et al. (2018) algorithm
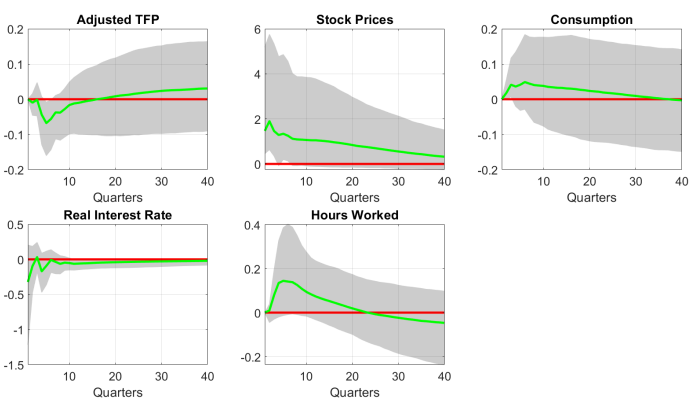

(d) Factor sign restrictions algorithm, with additional zero restrictions

Figure 2: This figure replicates the results in Beaudry et al. (2011), using a five variable $V A R$ for assessing the effects of an optimism shock. The sign-restricted impulse responses of the five variables are estimated using (a) the PFA algorithm of Mountford and Uhlig (2009); (b) the importance sampling approach of Arias et al. (2018); (c) the Gibbs sampler algorithm proposed in this paper; and (d) the algorithm of this paper, with additional zero restrictions in consumption and hours.

Panel (c) of Figure 2 shows the results from the factor sign restrictions algorithm using the same zero restriction on TFP and positive sign restriction on stock prices. The response of stock prices is not as pronounced as in Beaudry et al. (2011), and in general the responses for TFP, stock prices and real interest rate are equivalent to Arias et al. (2018). However, the responses of consumption and hours are still strongly different from zero, even though they have error bands and shapes that look much closer to those produced by the algorithm of Arias et al. (2018). Nevertheless, one aspect of the factor sign restrictions is that we can explicitly derive the implied fit to the VAR of imposing various restrictions. Therefore, we can explicitly test the premise of Arias 
et al. (2018) that consumption and hours are not affected by optimism shocks. Panel (d) in Figure 2 repeats estimation of the VAR using factor sign restrictions algorithm with additional zero restrictions in consumption and hours. The IRFs now look quantitatively and qualitatively closer to those in panel (b). Most importantly, we are able to test whether the model in panel (c) or (d) is supported by the data, that is, test whether the zero restrictions in consumption and hours. The DIC for the model without these restrictions is -13109.49 while the DIC for the model with the two zero restrictions is 15267.64. Thus, data evidence (which is conditional, of course, on our specific parametric likelihood specification and prior) suggests that the premise of Arias et al. (2018) - that optimism shocks do not affect consumption and hours - is correct.

\subsection{A (reasonably) large-scale VAR model for measuring financial shocks}

In this part of our real-data evaluation of the new algorithm we undertake the empirical exercise in Furlanetto et al. (forthcoming), who aim to measure various financial shocks to the US economy. Given computational restrictions, due to their use of the RubioRamírez et al. (2010) accept/reject algorithm, Furlanetto et al. (forthcoming) end up estimating a series of smaller VARs in order to sequentially measure and label interesting financial shocks, such as uncertainty, credit and housing. Before illustrating how to use our new algorithm to collectively measure all these shocks in one high-dimensional data setting, we first try to replicate their benchmark results. Among all VAR specifications, Furlanetto et al. (forthcoming) specify a baseline VAR specification with $p=5$ lags, using data on real GDP, consumer prices, interest rate, investment-to-output ratio, stock prices, and the external finance premium. ${ }^{16}$ All data are for the 1985Q1 - 2013Q2 period.

\footnotetext{
${ }^{16}$ The external finance premium is defined as the spread between yields on Baa rated bonds and the federal funds rate. Notice that the three variables that are not already expressed as rate, ratio, or spread (i.e. GDP, consumer prices, and stock prices), are transformed only using logarithms of the
} 
The online Appendix provides exact details of all series and transformations used, which in this case they are identical to those reported in Furlanetto et al. (forthcoming, Table 11).
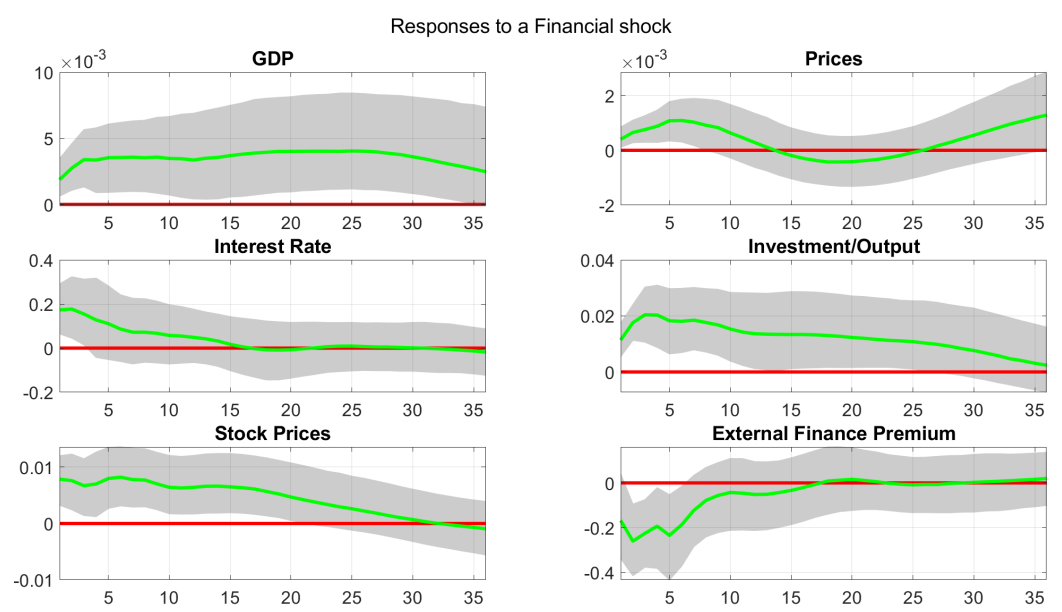

(a) Rubio-Ramírez et al. (2010) algorithm
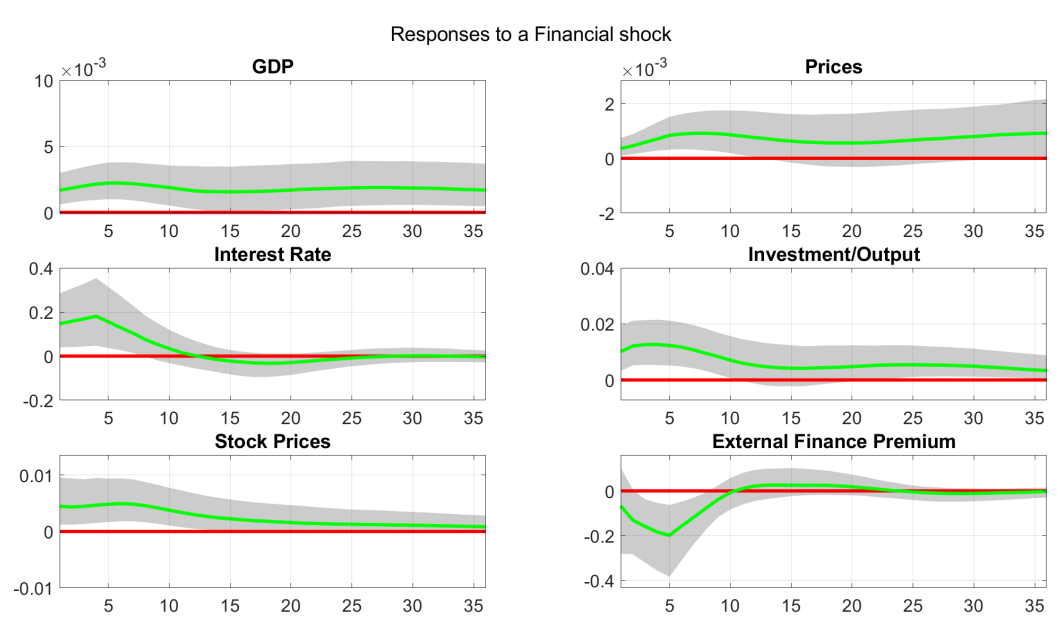

(b) Factor sign restrictions algorithm

Figure 3: This figure replicates the impulse response functions to a financial shock using the baseline specification of Furlanetto et al. (forthcoming). Panel (a) shows results based on the exact configuration of Furlanetto et al. (forthcoming, see Figure 1), using the algorithm of Rubio-Ramirez et al. (2010). Panel (b) replicates the same financial shock using the new sign restrictions algorithm.

levels and not growth rates - see the online Appendix for exact definitions, transformations, and data sources. Also note that these authors use a noninformative (uniform) prior, while estimation using our algorithm is implemented with the shrinkage horseshoe prior described in Section 2. 
Figure 3 shows the effects of a financial shock identified as a shock that causes GDP, consumer prices, stock prices, interest rate and the investment/output ratio to react positively contemporaneously. The sign of the spread is left unrestricted. Panel (a) replicates the impulse responses also shown in Figure 1 of Furlanetto et al. (forthcoming). Panel (b) shows the same responses produced by application of the new algorithm for sign restrictions. While there are obvious differences in how wide the bands of the IRFs are (due to the underlying assumptions about the VAR likelihood and the prior distributions), the results are qualitatively very similar and assuring that the new algorithm produces sensible results. Following up on the discussion in the previous section, it takes roughly four hours to obtain 2000 draws from the Furlanetto et al. (forthcoming) using their MATLAB code and exact numerical settings based on the Rubio-Ramírez et al. (2010) algorithm. In contrast, it takes less than five minutes to obtain 60,000 draws from our proposed Gibbs sampler (where out of these 60,000 draws we discard 10,000 and then save every $10^{\text {th }}$ draw, leading to 5,000 draws from the posterior of VAR parameters and impulse response functions).

We next proceed to demonstrate how the new algorithm can estimate one, largedimensional system in order to measure in one setting all the financial shocks that Furlanetto et al. (forthcoming) identify. The larger VAR that these authors specify has seven variables and six shocks: aggregate supply, aggregate demand, investment, housing, uncertainty, and credit. These authors do not identify a monetary shock using this larger VAR, possibly due to computational concerns. Here we attempt to use all available variables in Furlanetto et al. (forthcoming) to identify seven shocks, that is, the six shocks just listed plus a monetary shock. We also use additional measures of output, consumer prices, stock prices, interest rate, and credit spread, in order to enhance identification. We end up with a 15 -variable VAR with $p=5$ on the following variables: 1) real GDP; 2) prices (GDP deflator); 3) interest rate (3-month Tbill); 4) 
investment to output ratio; 5) stock prices (real S\&P500 prices); 6) credit spread (Baa minus Fed funds rate); 7) credit to real estate value ratio; 8) excess bond premium (EBP); 9) EBP to VIX ratio; 10) mortgage rate (30-year rates); 11) employment; 12) Federal funds rate; 13) core CPI; 14) stock prices 2 (real DJIA prices); and 15) credit spread 2 ("GZ" spread). The online Appendix has detailed definitions of these variables, transformations used, and sources.

Table 4 shows the signs imposed on each of the 15 variables in order to identify each of the seven structural shocks. This is a large matrix of restrictions, but our algorithm can handle computationally the task of 60,000 from the posterior of all parameters (including the structural matrix of contemporaneous shocks) in a matter of minutes. Like we did with the baseline VAR above, out of these 60,000 draws we discard 10,000 and we save every $10^{\text {th }}$ sample, resulting in 5,000 samples used to produce numerical results from this large model. The horseshoe prior also has a crucial role in estimating this model, as we have 1140 elements in $\boldsymbol{\Phi}$ and only 114 observations for each of the 15 endogenous variables. 
SHOCKS

\begin{tabular}{|c|c|c|c|c|c|c|c|}
\hline & Supply & Demand & Monetary & Investment & Housing & Uncertainty & Credit \\
\hline \multicolumn{8}{|c|}{ ORIGINAL VARIABLES IN FURLANETTO ET AL. (FORTHCOMING): } \\
\hline GDP & + & + & + & + & + & + & + \\
\hline prices & - & + & + & + & + & + & + \\
\hline interest rate & NA & + & - & + & + & + & + \\
\hline investment/output & NA & - & NA & + & + & + & + \\
\hline stock prices & + & $\mathrm{NA}$ & NA & - & + & + & + \\
\hline spread & NA & NA & NA & NA & NA & NA & NA \\
\hline credit/real estate value & NA & NA & NA & NA & - & + & + \\
\hline $\mathrm{EBP}$ & NA & NA & NA & NA & NA & - & - \\
\hline EBP/VIX & NA & $\mathrm{NA}$ & NA & NA & NA & + & - \\
\hline mortgage rates & NA & NA & NA & NA & NA & NA & - \\
\hline \multicolumn{8}{|c|}{ ADDITIONAL MEASURES OF OUTPUT, PRICES ETC.: } \\
\hline employment & + & + & + & + & + & + & + \\
\hline Federal funds rate & NA & + & - & + & + & + & + \\
\hline core prices & - & + & + & + & + & + & + \\
\hline stock prices 2 & + & NA & $\mathrm{NA}$ & - & + & + & + \\
\hline spread 2 & NA & NA & NA & NA & NA & NA & NA \\
\hline
\end{tabular}

Notes: Entries in this table show the restrictions imposed: + for positive sign; - for negative sign; NA for no restriction.

Table 4: Identified shocks and sign restrictions in the large, 15-variable VAR model

Figure 4 shows the impulse responses of the 15 endogenous variables to a credit shock. The green lines are posterior medians, and the shaded areas $68 \%$ bands. The magnitudes and shapes of the IRFs are consistent with the ones reported in Furlanetto et al. (forthcoming, Figure 7), despite the fact that in the case of variables such as GDP our IRFs are strongly different from zero. The most interesting feature of this figure is the effect of a credit shock on the two credit spread variables we used in the same VAR. Furlanetto et al. (forthcoming) use these spreads (plus an additional third spread we haven't included here) one at a time in their VAR in order to assess robustness of their results. These authors do not impose sign restrictions on the credit spread and 
they find that in their baseline specification this tends to be negative. In our case, the first credit spread variable has a strong negative contemporaneous response before subsequently moving to positive territory, while the second credit variable does not have a contemporaneous response different from zero and in subsequent period reacts positively. Such results show the important avenues for identifying various structural shocks that the new algorithm opens up: by using large information sets we can have the ability to identify several structural shocks in one setting, thus, making comparisons and testing of structural hypotheses more transparent. The online Appendix has figures of the impulse responses to the remaining identified six shocks in our 15-variable VAR setting. 

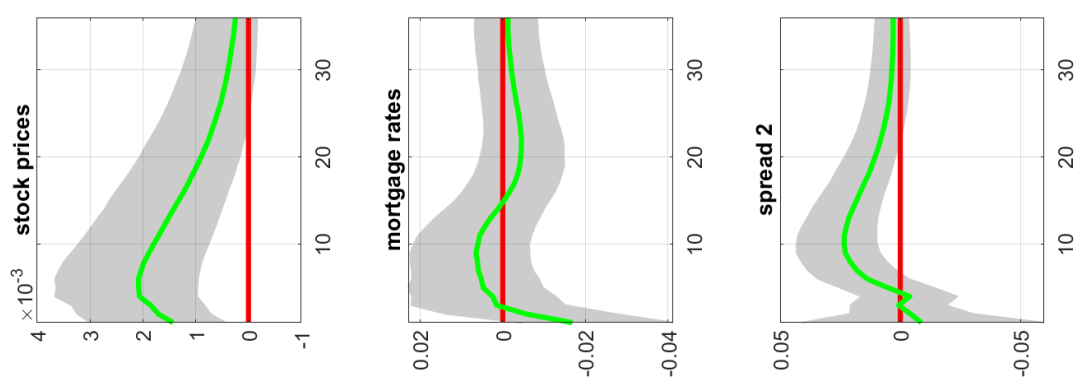

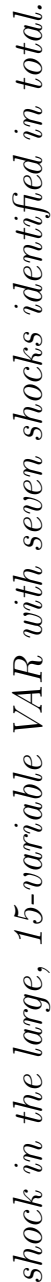
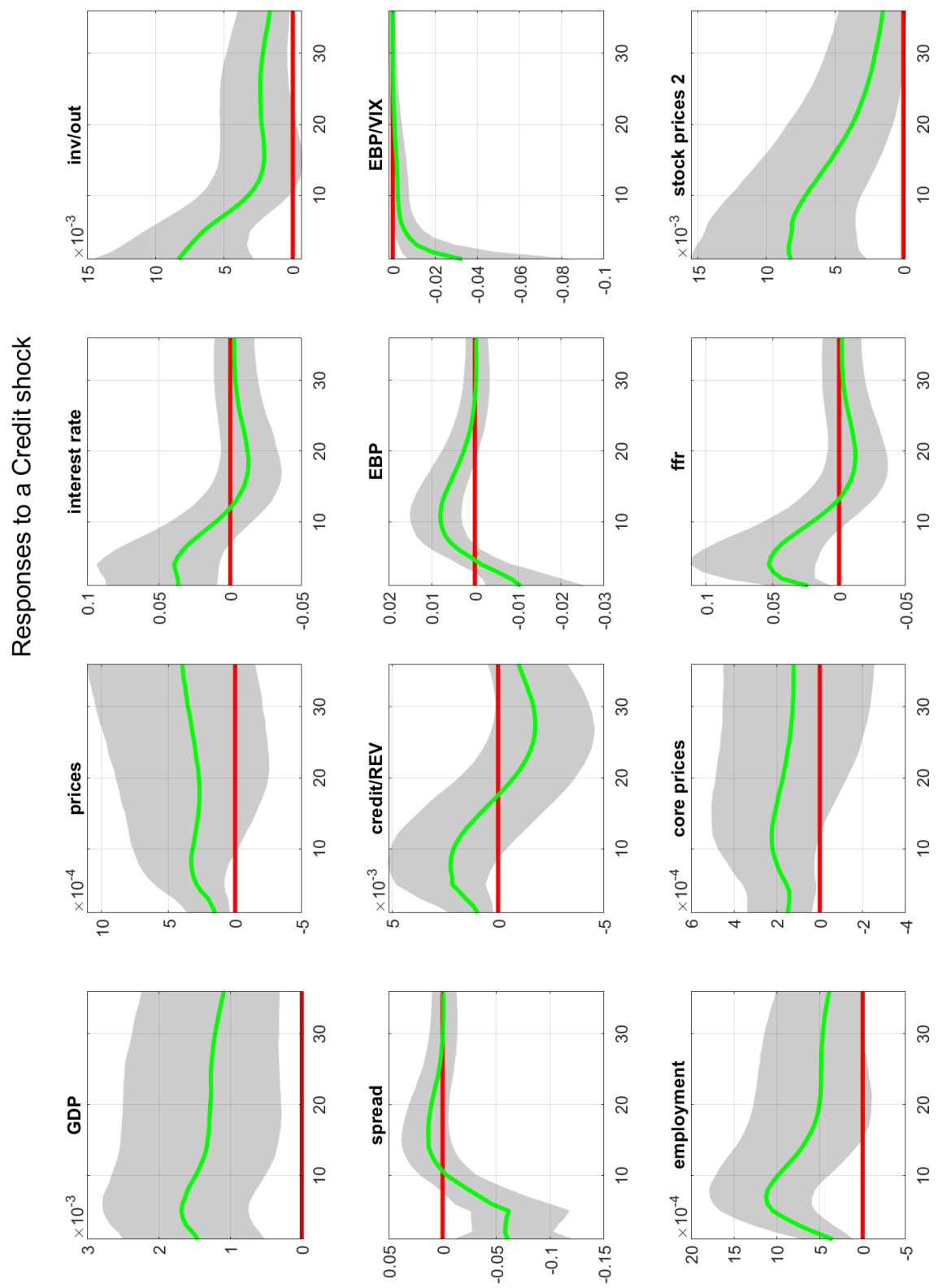


\section{References}

Adalid, R., D. Korobilis, And A. Musso (2019): "The Driving Forces of Credit Cycles in the Euro Area," mimeo, European Central Bank.

Ahmadi, P. A. And H. Uhlig (2015): "Sign Restrictions in Bayesian FaVARs with an Application to Monetary Policy Shocks," Working Paper 21738, National Bureau of Economic Research.

Anderson, T. W. And H. Rubin (1956): "Statistical Inference in Factor Analysis," in Proceedings of the Third Berkeley Symposium on Mathematical Statistics and Probability, Volume 5: Contributions to Econometrics, Industrial Research, and Psychometry, Berkeley, Calif.: University of California Press, 111-150.

Arias, J. E., D. Caldara, And J. F. Rubio-Ramírez (2019): "The Systematic Component of Monetary Policy in SVARs: An Agnostic Identification Procedure," Journal of Monetary Economics, 101, 1 - 13.

Arias, J. E., J. F. Rubio-Ramírez, And D. F. Waggoner (2018): "Inference Based on Structural Vector Autoregressions Identified With Sign and Zero Restrictions: Theory and Applications," Econometrica, 86, 685-720.

Armagan, A., D. B. Dunson, J. Lee, W. U. Bajwa, And N. Strawn (2013): "Posterior Consistency in Linear Models Under Shrinkage Priors," Biometrika, 100, 1011-1018.

BAI, J. (2003): "Inferential Theory for Factor Models of Large Dimensions," Econometrica, 71, 135-171.

Baumeister, C. And J. D. Hamilton (2015): "Sign Restrictions, Structural Vector Autoregressions, and Useful Prior Information," Econometrica, 83, 1963-1999. 
Beaudry, P., D. Nam, And J. Wang (2011): "Do Mood Swings Drive Business Cycles and is it Rational?" Working Paper 17651, National Bureau of Economic Research.

Bernanke, B. S. And A. S. Blinder (1992): "The Federal Funds Rate and the Channels of Monetary Transmission," The American Economic Review, 82, 901-921.

Bernanke, B. S., J. Boivin, And P. Eliasz (2005): "Measuring the Effects of Monetary Policy: A Factor-Augmented Vector Autoregressive (FAVAR) Approach*," The Quarterly Journal of Economics, 120, 387-422.

Bhattacharya, A., A. Chakraborty, And B. K. Mallick (2016): "Fast Sampling with Gaussian Scale Mixture Priors in High-Dimensional Regression," Biometrika, 103, 985-991.

Botev, Z. I. (2017): "The Normal Law Under linear Restrictions: Simulation and Estimation via Minimax Tilting," Journal of the Royal Statistical Society: Series B (Statistical Methodology), 79, 125-148.

Canova, F. And G. D. Nicoló (2002): "Monetary Disturbances Matter for Business Fluctuations in the G-7," Journal of Monetary Economics, 49, 1131 - 1159.

Canova, F. And F. J. PÉrez Forero (2015): "Estimating Overidentified, Nonrecursive, Time-Varying Coefficients Structural Vector Autoregressions," Quantitative Economics, 6, 359-384.

Carvalho, C. M., N. G. Polson, And J. G. Scott (2010): "The Horseshoe Estimator for Sparse Signals," Biometrika, 97, 465-480.

Furlanetto, F., F. Ravazzolo, and S. Sarferaz (forthcoming): "Identification of Financial Factors in Economic Fluctuations," The Economic Journal, Eearly View, doi:10.1111/ecoj.12520. 
Geweke, J. F. (1996): "Bayesian Inference for Linear Models Subject to Linear Inequality Constraints," in Modelling and Prediction Honoring Seymour Geisser, ed. by J. C. Lee, W. O. Johnson, and A. Zellner, New York, NY: Springer New York, $248-263$.

Ghosh, P., X. Tang, M. Ghosh, and A. Chakrabarti (2016): "Asymptotic Properties of Bayes Risk of a General Class of Shrinkage Priors in Multiple Hypothesis Testing Under Sparsity," Bayesian Anal., 11, 753-796.

Giacomini, R. And T. Kitagawa (2018): "Robust Bayesian Inference for SetIdentified Models," CeMMAP working papers CWP61/18, Centre for Microdata Methods and Practice, Institute for Fiscal Studies.

Giannone, D., M. Lenza, And G. E. Primiceri (2015): "Prior Selection for Vector Autoregressions," The Review of Economics and Statistics, 97, 436-451.

Gorodnichenko, Y. (2005): "Reduced-Rank Identification of Structural Shocks in VARs," SSRN working paper 590906, University of California, Berkeley.

Kastner, G., S. Frühwirth-Schnatter, And H. F. Lopes (2017): "Efficient Bayesian Inference for Multivariate Factor Stochastic Volatility Models," Journal of Computational and Graphical Statistics, 26, 905-917.

Kose, M. A., C. Otrok, And C. H. Whiteman (2003): "International Business Cycles: World, Region, and Country-Specific Factors," American Economic Review, 93, 1216-1239.

Kowal, D. R., D. S. Matteson, And D. Ruppert (2019): "Dynamic Shrinkage Processes," Journal of the Royal Statistical Society: Series B (Statistical Methodology), $81,781-804$. 
Lopes, H. F. And M. West (2004): "Bayesian Model Assessment in Factor Analysis," Statistica Sinica, 14, 41-67.

Matthes, C. And F. Schwartzman (2019): "What Do Sectoral Dynamics Tell Us About the Origins of Business Cycles?" Working Paper 19-9, Federal Reserve Bank of Richmond.

Mountford, A. And H. Uhlig (2009): "What Are the Effects of Fiscal Policy Shocks?" Journal of Applied Econometrics, 24, 960-992.

Neal, R. M. (2003): "Slice sampling," The Annals of Statistics, 31, 705-767.

PlagborG-Møller, M. (2019): "Bayesian Inference on Structural Impulse Response Functions," Quantitative Economics, 10, 145-184.

Ramey, V. (2016): "Chapter 2 - Macroeconomic Shocks and Their Propagation," in Handbook of Macroeconomics, ed. by J. B. Taylor and H. Uhlig, Elsevier, vol. 2, 71 162.

Rubio-Ramírez, J. F., D. F. Waggoner, and T. Zha (2010): "Structural Vector Autoregressions: Theory of Identification and Algorithms for Inference," The Review of Economic Studies, 77, 665-696.

Sims, C. A. AND T. ZhA (2006): "Were There Regime Switches in U.S. Monetary Policy?" American Economic Review, 96, 54-81.

Spiegelhalter, D. J., N. G. Best, B. P. Carlin, and A. Van Der Linde (2002): "Bayesian Measures of Model Complexity and Fit," Journal of the Royal Statistical Society: Series B (Statistical Methodology), 64, 583-639.

Stock, J. H. And M. W. Watson (2005a): "Implications of Dynamic Factor Models for VAR Analysis," Working Paper 11467, National Bureau of Economic Research. 
— (2005b): "Understanding Changes in International Business Cycle Dynamics," Journal of the European Economic Association, 3, 968-1006.

UhLIG, H. (2005): "What are the effects of monetary policy on output? Results from an agnostic identification procedure," Journal of Monetary Economics, 52, 381 - 419.

van der Pas, S. L., B. J. K. Kleijn, And A. W. van Der VaArT (2014): "The horseshoe estimator: Posterior concentration around nearly black vectors," Electronic Journal of Statistics, 8, 2585-2618.

Waggoner, D. F. And T. Zha (2003): "A Gibbs sampler for structural vector autoregressions," Journal of Economic Dynamics and Control, 28, 349 - 366. 


\title{
Online Appendix to "Sign restrictions in high-dimensional vector autoregressions"
}

\author{
Dimitris Korobilis \\ University of Glasgow
}

\section{A Full Gibbs sampler for VARs with sign restrictions}

The Gibbs sampler presented in the main paper is a quite accurate description of the core algorithmic steps required in order to estimate the VAR model with factor structure in the residuals. Nevertheless, in order to produce empirical and other results, we have relied on the hierarchical horseshoe prior of Carvalho et al. (2010), two fast algorithms from drawing from the Normal (Bhattacharya et al., 2016) and truncated Normal (Botev, 2017) distributions, respectively, and the slice sampler of Neal (2003) in order to update the horseshoe prior parameters. Therefore, it is important to rewrite the Gibbs sampling algorithm in full, and give further explanations about the three enhancements that guarantee a fast and reliable algorithm in high dimensions.

We repeat the full prior specification, which now includes the hierarchical horseshoe 
prior on $\phi_{i}$. The priors for the $i^{t h}$ VAR equation, $i=1, \ldots, n$ is:

$$
\begin{aligned}
\boldsymbol{\phi}_{i} \mid \sigma_{i}^{2}, \tau_{i}^{2}, \mathbf{\Psi}_{i}^{2} & \sim N_{k}\left(\mathbf{0}, \sigma_{i}^{2} \tau_{i}^{2} \mathbf{\Psi}_{i}^{2}\right), \quad \mathbf{\Psi}_{i}^{2}=\operatorname{diag}\left(\psi_{i, 1}^{2}, \ldots, \psi_{i, k}^{2}\right), \\
\psi_{i, j} & \sim \operatorname{Cauchy}^{+}(0,1), j=1, \ldots, k, \\
\tau_{i} & \sim \operatorname{Cauchy}^{+}(0,1), \\
\boldsymbol{\Lambda}_{i j} & \sim\left\{\begin{array}{ll}
N\left(0, \underline{h}_{i j}\right) I\left(\Lambda_{i j}>0\right), & \text { if } S_{i j}=1, \\
N\left(0, \underline{h}_{i j}\right) I\left(\Lambda_{i j}<0\right), & \text { if } S_{i j}=-1, \\
\delta_{0}\left(\boldsymbol{\Lambda}_{i j}\right), & \text { if } S_{i j}=0, \\
N\left(0, \underline{h}_{i j}\right), & \text { otherwise, }
\end{array} \quad, \ldots, r,\right. \\
\mathbf{f}_{t} & \sim N_{r}(\mathbf{0}, \mathbf{I}), \\
\sigma_{i}^{2} & \sim \text { inv }-\operatorname{Gamma}\left(\underline{\rho}_{i}, \underline{\kappa}_{i}\right),
\end{aligned}
$$

where we set $\underline{h}_{i j}=4, \underline{\rho}_{i}=1$ and $\underline{\kappa}_{i}=0.01$.

Under these priors, the full factor sign restrictions algorithm takes the following form

\section{Factor sign restrictions (FSR) algorithm}

1. Sample $\phi_{i}$ for $i=1, \ldots, n$ from

$$
\boldsymbol{\phi}_{i} \mid \boldsymbol{\Sigma}, \boldsymbol{\Lambda}, \mathbf{f}, \mathbf{y} \sim N_{k}\left(\overline{\mathbf{V}}_{i}\left(\sum_{t=1}^{T} \sigma_{i}^{-2} \mathbf{x}_{t}^{\prime} \widetilde{\mathbf{y}}_{i t}\right), \overline{\mathbf{V}}_{i}\right)
$$

where $\tilde{\mathbf{y}}_{i t}=\mathbf{y}_{i t}-\boldsymbol{\Lambda}_{i} \mathbf{f}_{t}$ and $\overline{\mathbf{V}}_{i}^{-1}=\left(\underline{\mathbf{V}}_{i}^{-1}+\sum_{t=1}^{T} \sigma_{i}^{-2} \mathbf{x}_{t}^{\prime} \mathbf{x}_{t}\right)$. We use the efficient sampler of Bhattacharya et al. (2016) in order to sample these elements.

2. Sample $\psi_{i j}$ using slice sampling (Neal, 2003)

a. Set $\eta_{i j}=1 / \psi_{i j}^{2}$ using the last available sample of $\psi_{i j}^{2}$. 
b. Sample a random variable $u$ from

$$
u \mid \eta_{i j} \sim \text { Uniform }\left(0, \frac{1}{1+\eta_{i j}}\right)
$$

c. Sample $\eta_{i j}$ from

$$
\eta_{i j} \sim e^{\frac{\phi_{i j}^{2}}{2 \sigma_{i}^{2}} \eta_{i j}} I\left(\frac{u}{1-u}>\eta_{i j}\right)
$$

and set $\psi_{i j}=1 / \sqrt{\eta_{i j}}$.

3. Sample $\tau_{i}$ using slice sampling (Neal, 2003)

a. Set $\xi_{i}=1 / \tau_{i}^{2}$ using the last available sample of $\tau_{i}^{2}$.

b. Sample a random variable $u$ from

$$
v \mid \xi_{i j} \sim \text { Uniform }\left(0, \frac{1}{1+\xi_{i j}}\right)
$$

c. Sample $\xi_{i}$ from

$$
\xi_{i} \sim \gamma\left((k+1) / 2, v \frac{2 \sigma^{2}}{\sum\left(\frac{\phi_{i j}}{\psi_{i j}}\right)^{2}}\right)
$$

where $\gamma(\bullet)$ is the lower incomplete gamma function, and set $\tau_{i}=1 / \sqrt{\xi_{i}}$

4. Sample $\boldsymbol{\Lambda}_{i j}$ from univariate conditional posteriors (Geweke, 1996) of the form

$$
\boldsymbol{\Lambda}_{i j} \mid \boldsymbol{\Lambda}_{-i j} \boldsymbol{\Phi}, \boldsymbol{\Sigma}, \mathbf{f}, \mathbf{y} \sim T N_{\left(\mathbf{a}_{i j}, \mathbf{b}_{i j}\right)}\left(\bar{\lambda}_{i j}-\bar{h}_{i j} \sum_{l \neq j} \bar{h}_{i l}^{-1}\left(\boldsymbol{\Lambda}_{i l}-\bar{\lambda}_{i l}\right), \bar{h}_{i j}\right)
$$

where $\bar{\lambda}_{i j}$ and $\bar{h}_{i j}$ denote the $i j^{\text {th }}$ elements of the joint posterior mean and variance, respectively, of $\boldsymbol{\Lambda}_{i}$. The joint posterior variance is $\overline{\mathbf{H}}^{-1}=\left(\underline{\mathbf{H}}^{-1}+\sum_{t=1}^{T} \sigma_{i}^{-2} \mathbf{f}_{t}^{\prime} \mathbf{f}_{t}\right)$ and the joint posterior mean is $\overline{\mathbf{H}}\left(\sum_{t=1}^{T} \sigma_{i}^{-2} \mathbf{f}_{t}^{\prime} \widehat{\mathbf{y}}_{i t}\right)$ with $\widehat{\mathbf{y}}_{i t} \equiv \boldsymbol{\varepsilon}_{i t}=\mathbf{y}_{i t}-\phi_{i} \mathbf{x}_{t}$. Here 
$T N_{\left(\mathbf{a}_{i j}, \mathbf{b}_{i j}\right)}(\bullet)$ denotes the univariate truncated Normal distribution with bounds:

$$
\left(\mathbf{a}_{i j}, \mathbf{b}_{i j}\right)= \begin{cases}(-\infty, 0) & \text { if } S_{i j}=-1 \\ (0, \infty) & \text { if } S_{i j}=1 \\ (0,0) & \text { if } S_{i j}=0 \\ (-\infty, \infty) & \text { otherwise }\end{cases}
$$

We use the efficient univariate truncated Normal generator provided by Botev (2017) in order to sample these elements.

5. Sample $\mathbf{f}_{t}$ for $t=1, \ldots, T$ from

$$
\mathbf{f}_{t} \mid \mathbf{\Lambda}, \boldsymbol{\Sigma}, \mathbf{\Phi}, \mathbf{y} \sim N\left(\overline{\mathbf{G}}\left(\boldsymbol{\Lambda} \Sigma^{-1} \widehat{\mathbf{y}}_{t}\right), \overline{\mathbf{G}}\right)
$$

where $\overline{\mathbf{G}}^{-1}=\left(\mathbf{I}_{r}+\boldsymbol{\Lambda}^{\prime} \boldsymbol{\Sigma} \boldsymbol{\Lambda}\right)$. Post-process the draws of the $T \times r$ matrix $\mathbf{f}=\left(\mathbf{f}_{1}, \ldots, \mathbf{f}_{T}\right)^{\prime}$ such that its $r$ columns (corresponding to structural shocks) are uncorrelated and standardized to unit variance. This is done by applying first the Gram-Schmidt procedure and subsequently dividing each column of $\mathbf{f}$ with its standard deviation.

6. Sample $\sigma_{i}^{2}$ for $i=1, \ldots, n$ from

$$
\sigma_{i}^{2} \mid \mathbf{\Lambda}, \mathbf{f}, \boldsymbol{\Phi}, \mathbf{y} \sim i n v-G a m m a\left(\frac{T}{2}+\underline{\rho}_{i},\left[\underline{\kappa}_{i}^{-1}+\sum_{t=1}^{T}\left(\mathbf{y}_{i t}-\phi_{i} \mathbf{x}_{t}-\boldsymbol{\Lambda}_{i} \mathbf{f}_{t}\right)^{\prime}\left(\mathbf{y}_{i t}-\phi_{i} \mathbf{x}_{t}-\boldsymbol{\Lambda}_{i} \mathbf{f}_{t}\right)\right]^{-1}\right)
$$




\section{B Data Appendix}

\section{B.1 Data for the simulation study}

The data used in the simulation exercise are an augmented version of the data used in Arias et al. (2019). The measure of commodity prices they provide comes from Global Financial Data, while all remaining variables come originally from St Louis' Federal Reserve Economic Data (FRED; https://fred.stlouisfed.org/). Monthly GDP and GDP deflator are constructed using interpolation, and the reader is referred to the data supplement of Arias et al. (2019) for more information. The variables these authors use are augmented with stock prices, M1, unemployment rate, industrial production, employment, consumer prices (total), core consumer prices (total, less food and energy) and personal consumption deflator.

All variable mnemonics, short descriptions, and sources are visible in Table B1. The last column (Tcode) refers to the stationarity transformation used for each variable, namely 1: levels, and 5: first differences of logarithm (growth rates). 
Table B1: Data used in Monte Carlo exercise

\begin{tabular}{lllc}
\hline Mnemonic & Description & Source & Tcode \\
\hline \hline GDPC1 & Monthly real GDP & Arias et al. (2019) & 5 \\
GDPDEFL & Monthly GDP deflator & Arias et al. (2019) & 5 \\
FEDFUNDS & Fed funds rate & Arias et al. (2019) & 1 \\
CPRINDEX & Commodity price index & Arias et al. (2019) & 5 \\
TRARR & Total reserves & Arias et al. (2019) & 5 \\
BOGNONBR & Nonborrowed reserves & Arias et al. (2019) & 5 \\
'GSPC & S\&P 500 prices & Yahoo! Finance & 5 \\
M1REAL & Real M1 money stock & from FRED (M1REAL) & 5 \\
UNRATE & Unemployment rate & from FRED (UNRATE) & 1 \\
INDPRO & Industrial production index, all industries & from FRED (INDPRO) & 5 \\
PAYEMS & Employment, total & from FRED (PAYEMS) & 5 \\
CPIAUSL & Consumer price index, all items & from FRED (CPIAUSL) & 5 \\
CPILFESL & Core CPI & from FRED (CPILFESL) & 5 \\
PCEPILFE & Core PCE deflator & from FRED (PCEPILFE) & 5 \\
\hline
\end{tabular}

\section{B.2 Data used for the large-scale VAR model for the US}

The full list of variables is shown in Table B2 and they pertain to the sample 1985Q1 - 2013Q2. The original mortgage rate used by Furlanetto et al. (forthcoming) was only available after 1990Q1, and for that reason it has been replaced by the 30-year mortgage rate provided by FRED (contemporaneous correlation between the two series is 0.9967 ). All remaining series used in the empirical exercise are exactly those described in Table 11 of Furlanetto et al. (forthcoming), augmented with a few additional measures of output, consumer prices, and stock prices. The fifteen variables used in the large VAR can be seen in the first column of Table 4. In this list stock prices refers to S\&P500 while stock prices 2 refers to Dow Jones Industrial Average. Similarly, spread refers to the 
Baa minus fed funds rate spread, while spread 2 refers to the GZ credit spread.

Table B2: Data used in the empirical exercise

\begin{tabular}{|c|c|c|}
\hline Variable & Description & Source \\
\hline GDP & Log of real GNP/GDP & Federal Reserve Bank of Philadelphia \\
\hline GDP deflator & Log of price index for GNP/GDP & Federal Reserve Bank of Philadelphia \\
\hline Interest rate & 3-month treasury bill & Federal Reserve Bank of St. Louis \\
\hline Investment & Log of real gross private domestic investment & Federal Reserve Bank of St. Louis \\
\hline Stock prices & Log of real S\&P 500 & Yahoo! Finance \\
\hline Total credit & Log of loans to non-financial private sector & $\begin{array}{l}\text { Board of Governors of the Federal Reserve } \\
\text { System }\end{array}$ \\
\hline Mortgages & $\begin{array}{l}\text { Log of home mortgages of households and } \\
\text { non-profit organizations }\end{array}$ & $\begin{array}{l}\text { Board of Governors of the Federal Reserve } \\
\text { System }\end{array}$ \\
\hline Real estate value & $\begin{array}{l}\text { Log of real estate at market value of } \\
\text { households and non-profit organizations }\end{array}$ & $\begin{array}{l}\text { Board of Governors of the Federal Reserve } \\
\text { System }\end{array}$ \\
\hline Corporate bond yield & Moody's baa corporate bond yield & Federal Reserve Bank of St. Louis \\
\hline Federal funds rate & Federal funds rate & Federal Reserve Bank of St. Louis \\
\hline GZ credit spread & $\begin{array}{l}\text { Senior unsecured corporate bond spreads } \\
\text { (non-financial firms) }\end{array}$ & Gilchrist and Zakrajšek $(2012)^{a}$ \\
\hline $\mathrm{EBP}$ & Excess bond premium & Gilchrist and Zakrajšek $(2012)^{a}$ \\
\hline VIX & Stock market volatility index & Bloom $(2009)^{b}$ \\
\hline Mortgage rates & $\begin{array}{l}\text { Home mortgages, fixed } 30 Y R \text {, Effective } \\
\text { interest rate }\end{array}$ & Federal Reserve Bank of St. Louis \\
\hline Employment & Log of total nonfarm employment & Federal Reserve Bank of Philadelphia \\
\hline Core prices & Log of core consumer price index & Federal Reserve Bank of Philadelphia \\
\hline Stock prices 2 & Log of real DJIA & Yahoo! Finance \\
\hline
\end{tabular}

a Gilchrist, Simon, and Egon Zakrajšek (2012), Credit Spreads and Business Cycle Fluctuations. American Economic Review, 102 (4): 1692-1720.

${ }^{b}$ Bloom, Nicholas (2009), The Impact of Uncertainty Shocks. Econometrica, 77: 623-685. 


\section{Additional results}

\section{C.1 Additional results for the first Monte Carlo exercise}
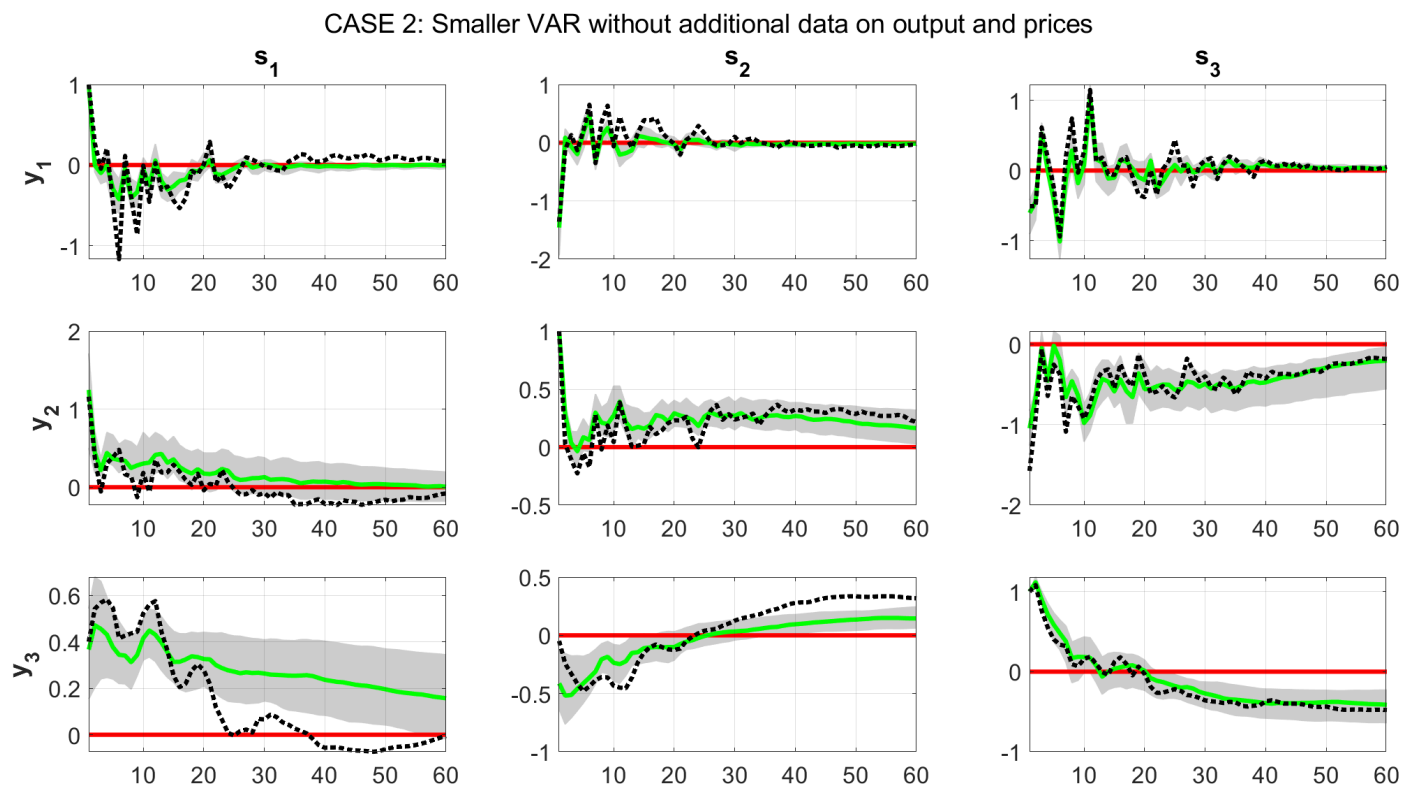

Figure C1: Impulse response functions of the first three artificially generated variables (denoted as $y_{1}, y_{2}, y_{3}$ ) in response to the three identified shocks (denoted as $s_{1}, s_{2}, s_{3}$ ) in model C2 (misspecified VAR dimension). The green solid lines show the posterior median IRFs over the 500 Monte Carlo iterations, and the gray shaded areas their associated 90\% bands. The true IRFs based on the DGP are shown using the black dashed lines. 


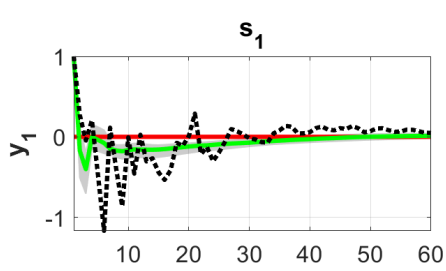

CASE 3: Misspecification of lag structure
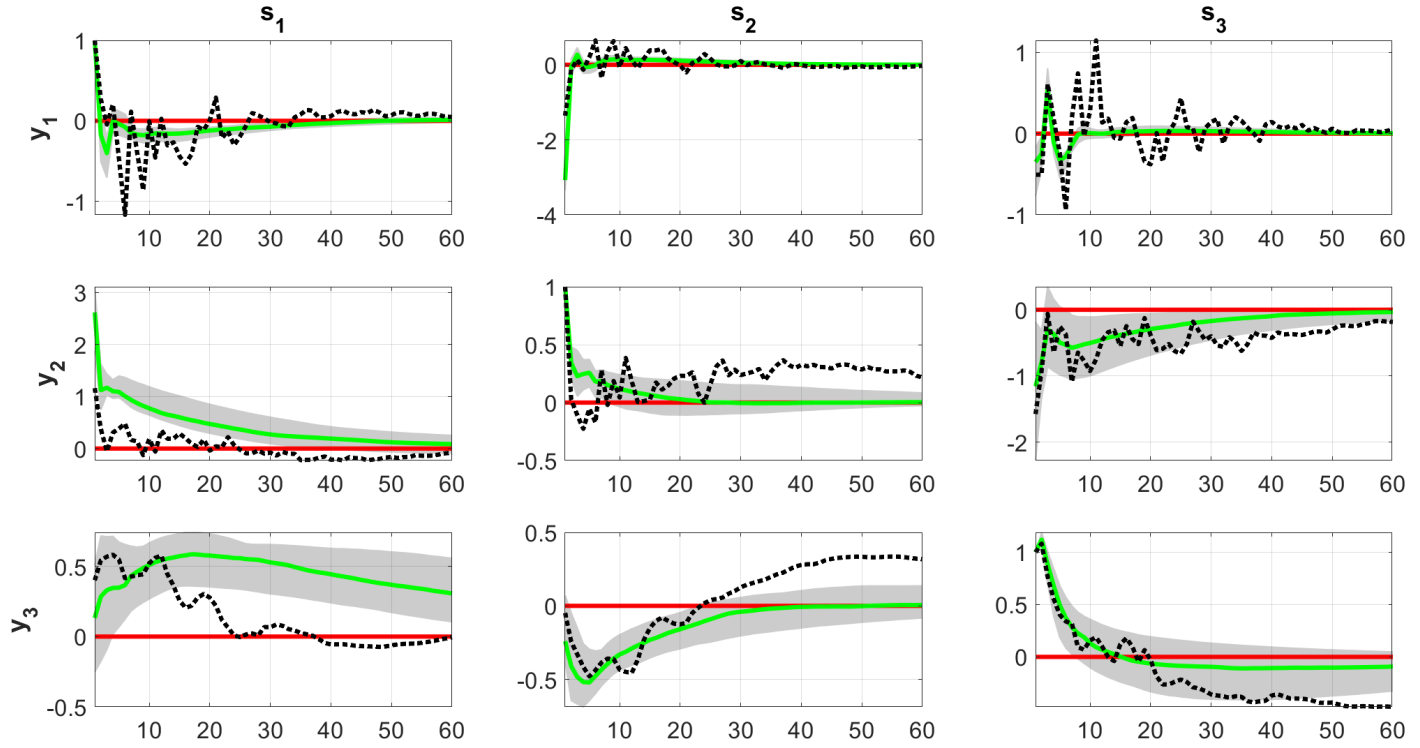

Figure C2: Impulse response functions of the first three artificially generated variables (denoted as $\left.y_{1}, y_{2}, y_{3}\right)$ in response to the three identified shocks (denoted as $s_{1}, s_{2}, s_{3}$ ) in model C3 (misspecified number of lags). The green solid lines show the posterior median IRFs over the 500 Monte Carlo iterations, and the gray shaded areas their associated 90\% bands. The true IRFs based on the DGP are shown using the black dashed lines. 

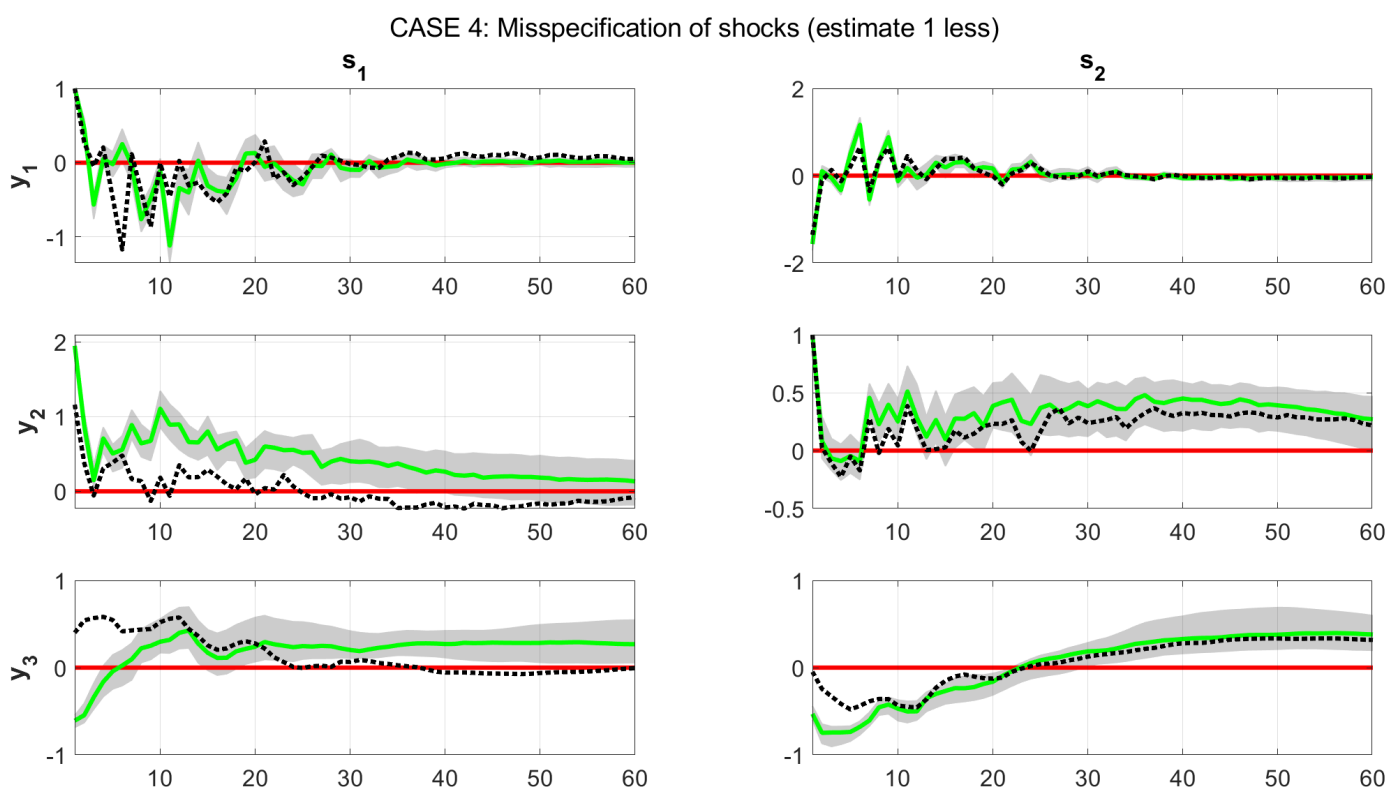

Figure C3: Impulse response functions of the first three artificially generated variables (denoted as $y_{1}, y_{2}, y_{3}$ ) in response to the three identified shocks (denoted as $s_{1}, s_{2}, s_{3}$ ) in model C4 (misspecified number of shocks - one less). The green solid lines show the posterior median IRFs over the 500 Monte Carlo iterations, and the gray shaded areas their associated 90\% bands. The true IRFs based on the DGP are shown using the black dashed lines. 

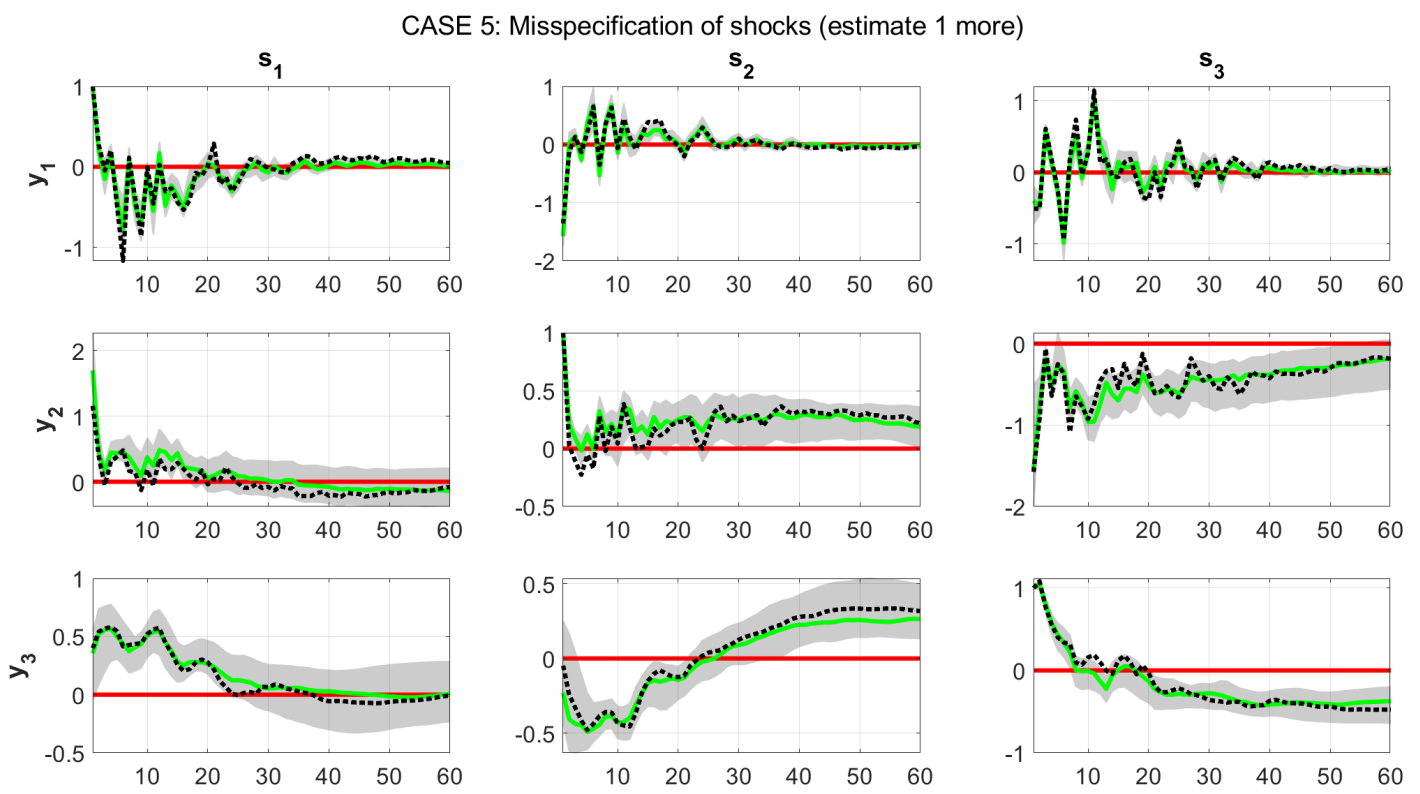

Figure C4: Impulse response functions of the first three artificially generated variables (denoted as $\left.y_{1}, y_{2}, y_{3}\right)$ in response to the three identified shocks (denoted as $s_{1}, s_{2}, s_{3}$ ) in model C5 (misspecified number of shocks - one more). The green solid lines show the posterior median IRFs over the 500 Monte Carlo iterations, and the gray shaded areas their associated 90\% bands. The true IRFs based on the DGP are shown using the black dashed lines. 


\section{C.2 Additional results for the baseline VAR of Furlanetto et al. (forthcoming)}

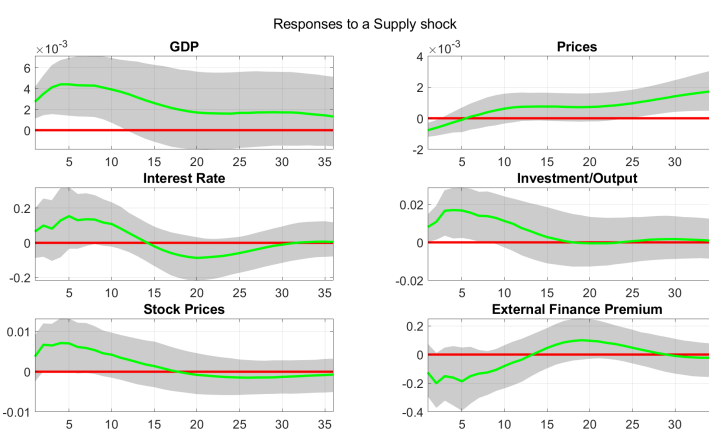

(a) Furlanetto et al. (forthcoming) algorithm

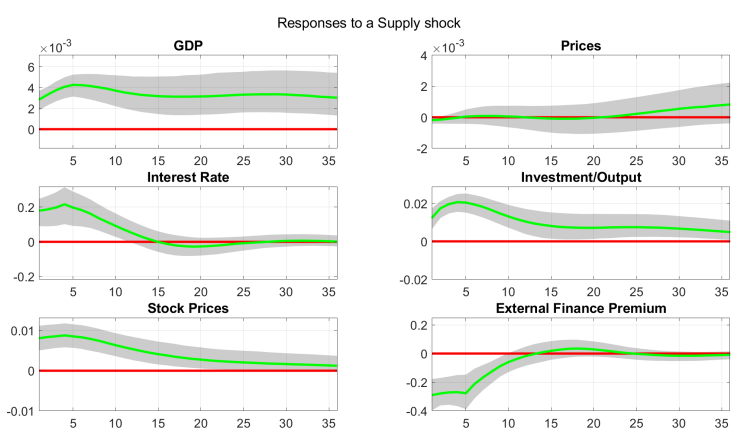

(b) Factor sign restrictions algorithm

Figure C5: This figure replicates the impulse response functions to an aggregate supply shock using the baseline specification of Furlanetto et al. (forthcoming). Panel (a) shows results based on the exact configuration of Furlanetto et al. (forthcoming, see Figure 1), using the algorithm of Rubio-Ramirez et al. (2010). Panel (b) replicates the same financial shock using the new sign restrictions algorithm.

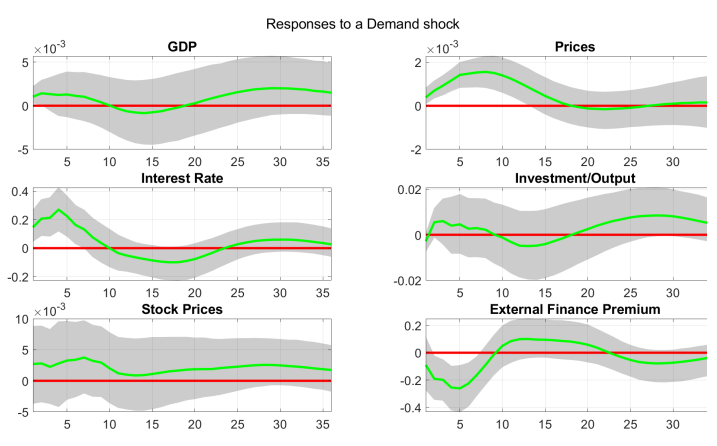

(a) Furlanetto et al. (forthcoming) algorithm

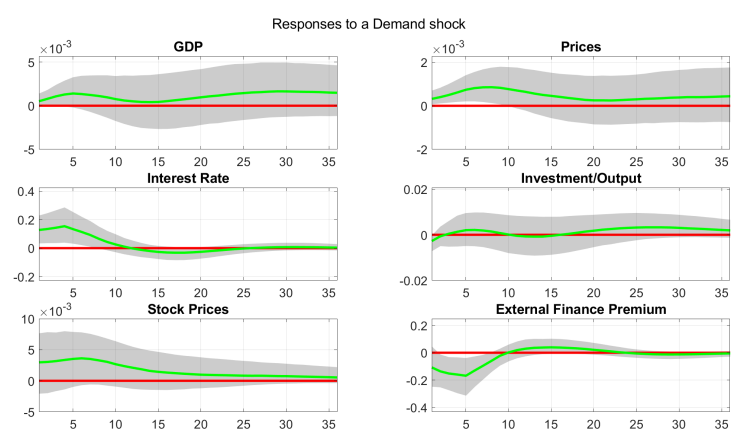

(b) Factor sign restrictions algorithm

Figure C6: This figure replicates the impulse response functions to an aggregate demand shock using the baseline specification of Furlanetto et al. (forthcoming). Panel (a) shows results based on the exact configuration of Furlanetto et al. (forthcoming, see Figure 1), using the algorithm of Rubio-Ramirez et al. (2010). Panel (b) replicates the same financial shock using the new sign restrictions algorithm. 


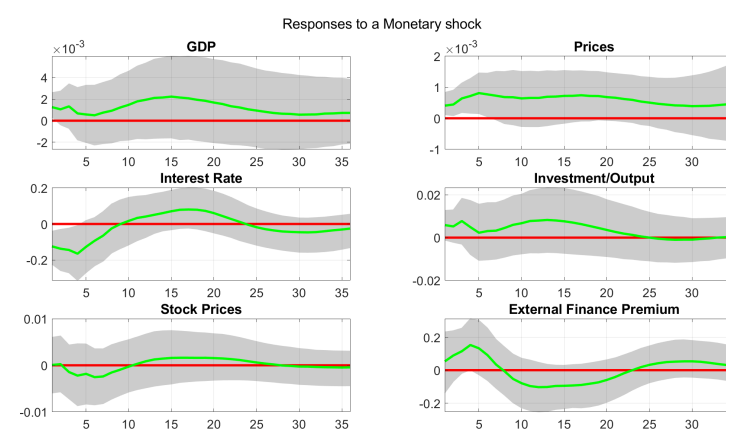

(a) Furlanetto et al. (forthcoming) algorithm

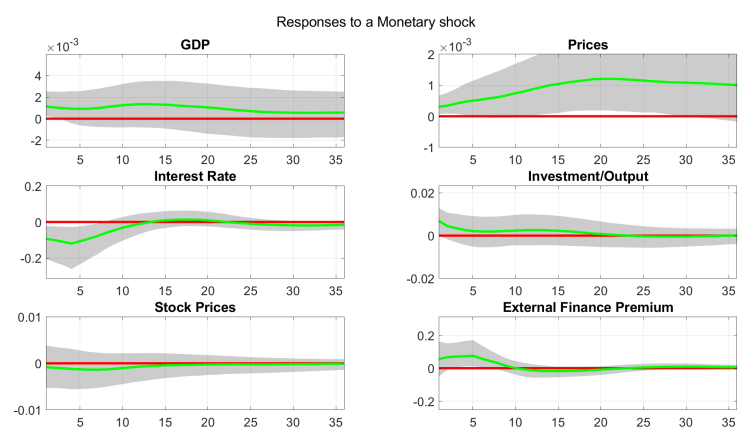

(b) Factor sign restrictions algorithm

Figure C7: This figure replicates the impulse response functions to a monetary policy shock using the baseline specification of Furlanetto et al. (forthcoming). Panel (a) shows results based on the exact configuration of Furlanetto et al. (forthcoming, see Figure 1), using the algorithm of Rubio-Ramirez et al. (2010). Panel (b) replicates the same financial shock using the new sign restrictions algorithm.

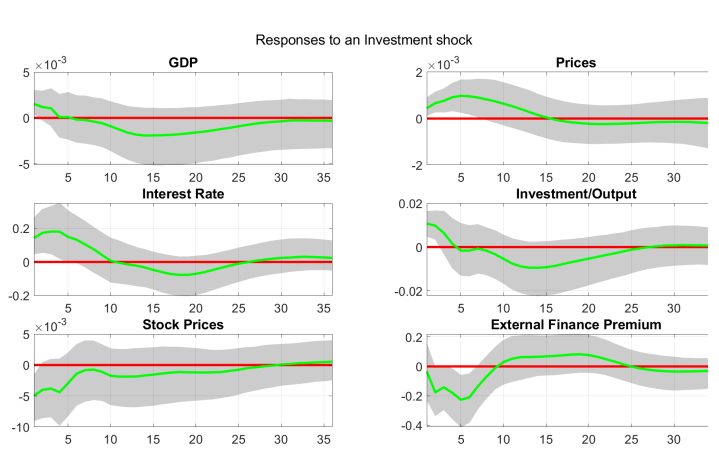

(a) Furlanetto et al. (forthcoming) algorithm

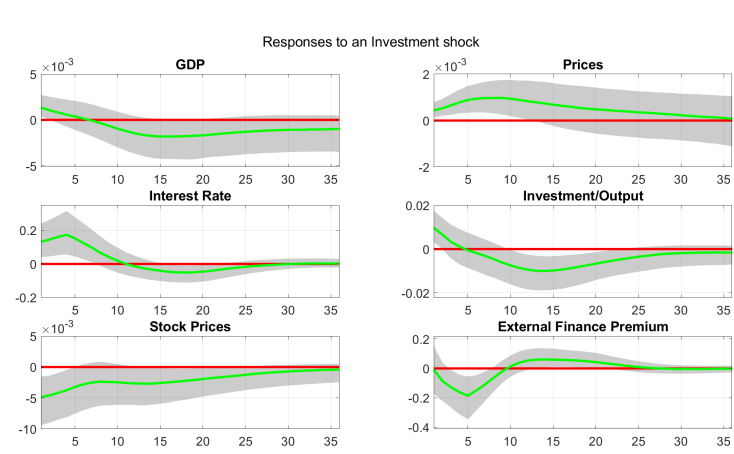

(b) Factor sign restrictions algorithm

Figure C8: This figure replicates the impulse response functions to an investment shock using the baseline specification of Furlanetto et al. (forthcoming). Panel (a) shows results based on the exact configuration of Furlanetto et al. (forthcoming, see Figure 1), using the algorithm of Rubio-Ramirez et al. (2010). Panel (b) replicates the same financial shock using the new sign restrictions algorithm.

\section{C.3 Additional impulse responses from the large-scale, 15-variable VAR}



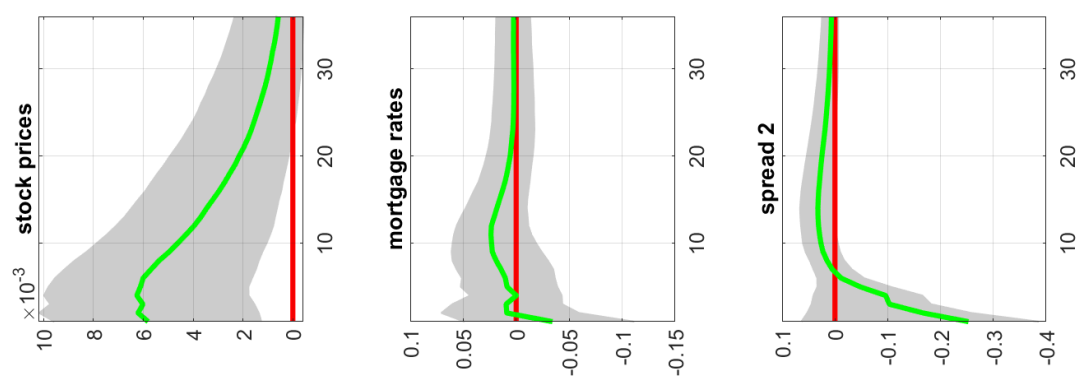

$\frac{5}{\frac{5}{0}}$
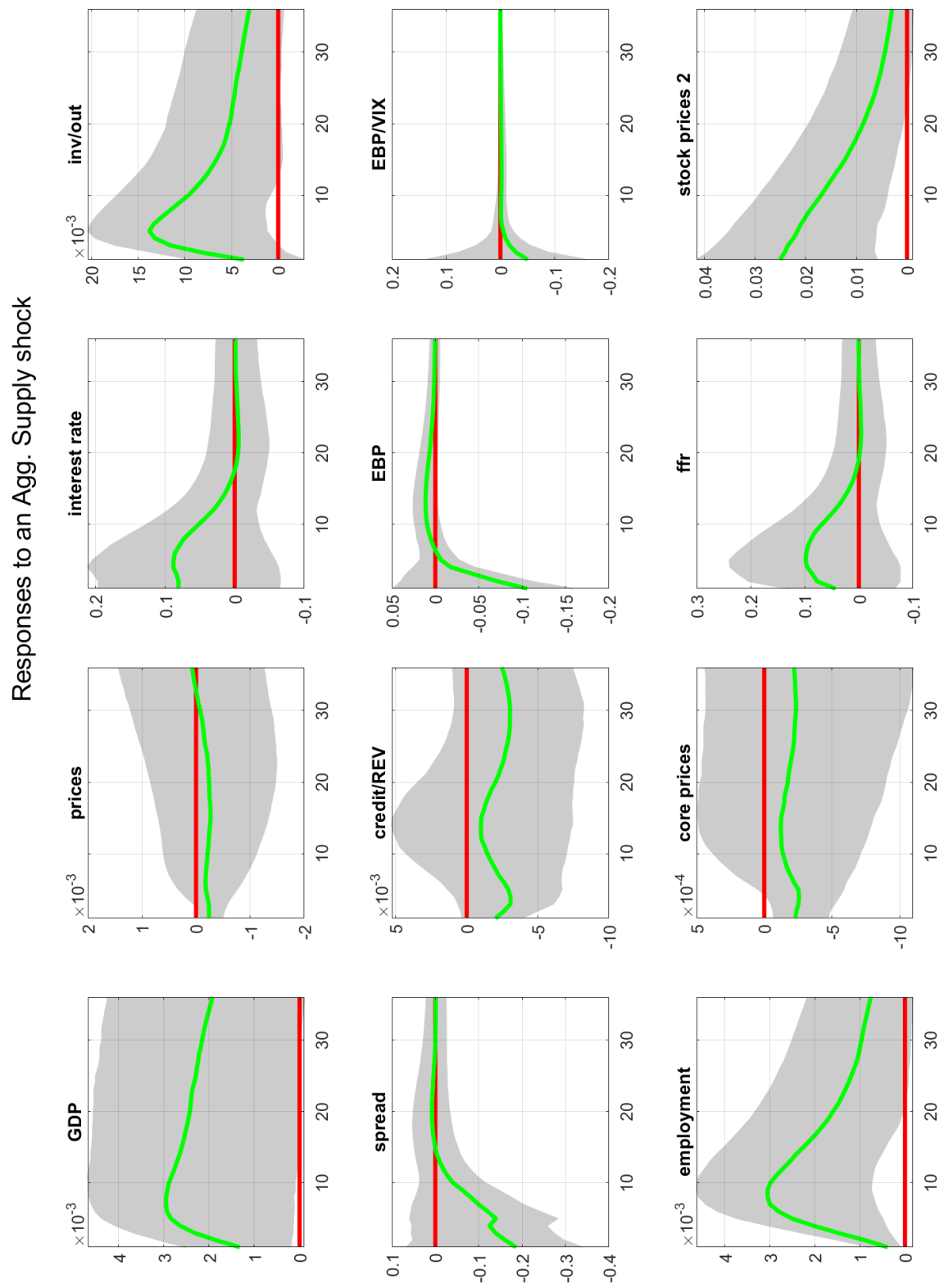

กี

$\frac{\sqrt{3}}{\sqrt[\pi]{3}}$

$\frac{\pi}{5}$

$\frac{1}{5}$
है
5
1
1

है

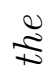

.

कू

న్తి

ث্

है

ह

인

芯

0
है
है
के

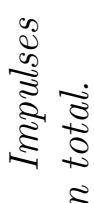

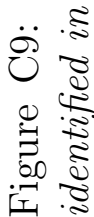



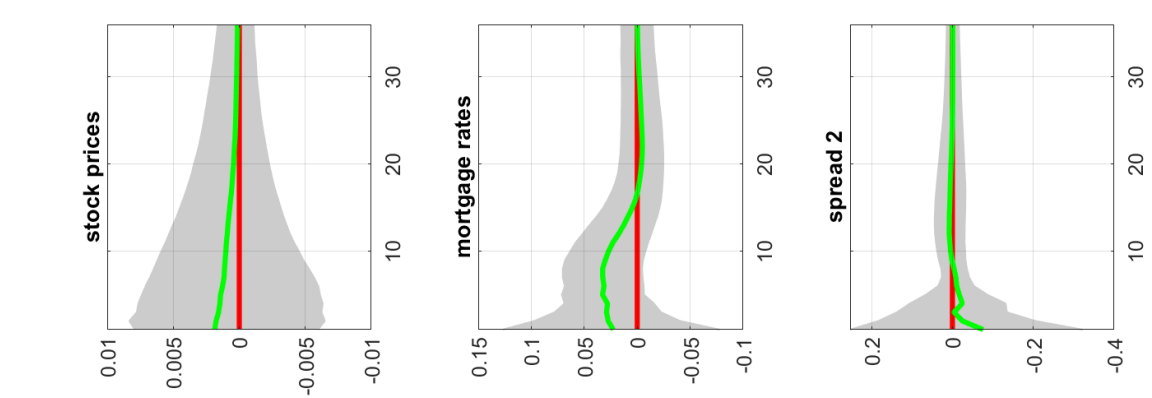

$\frac{5}{3}$
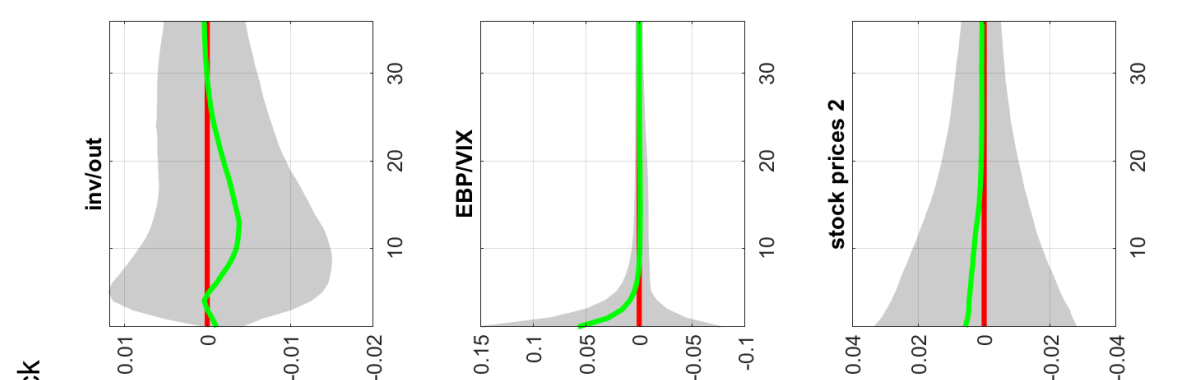

อิ

$\frac{\sqrt{3}}{\sqrt[\pi]{n}}$
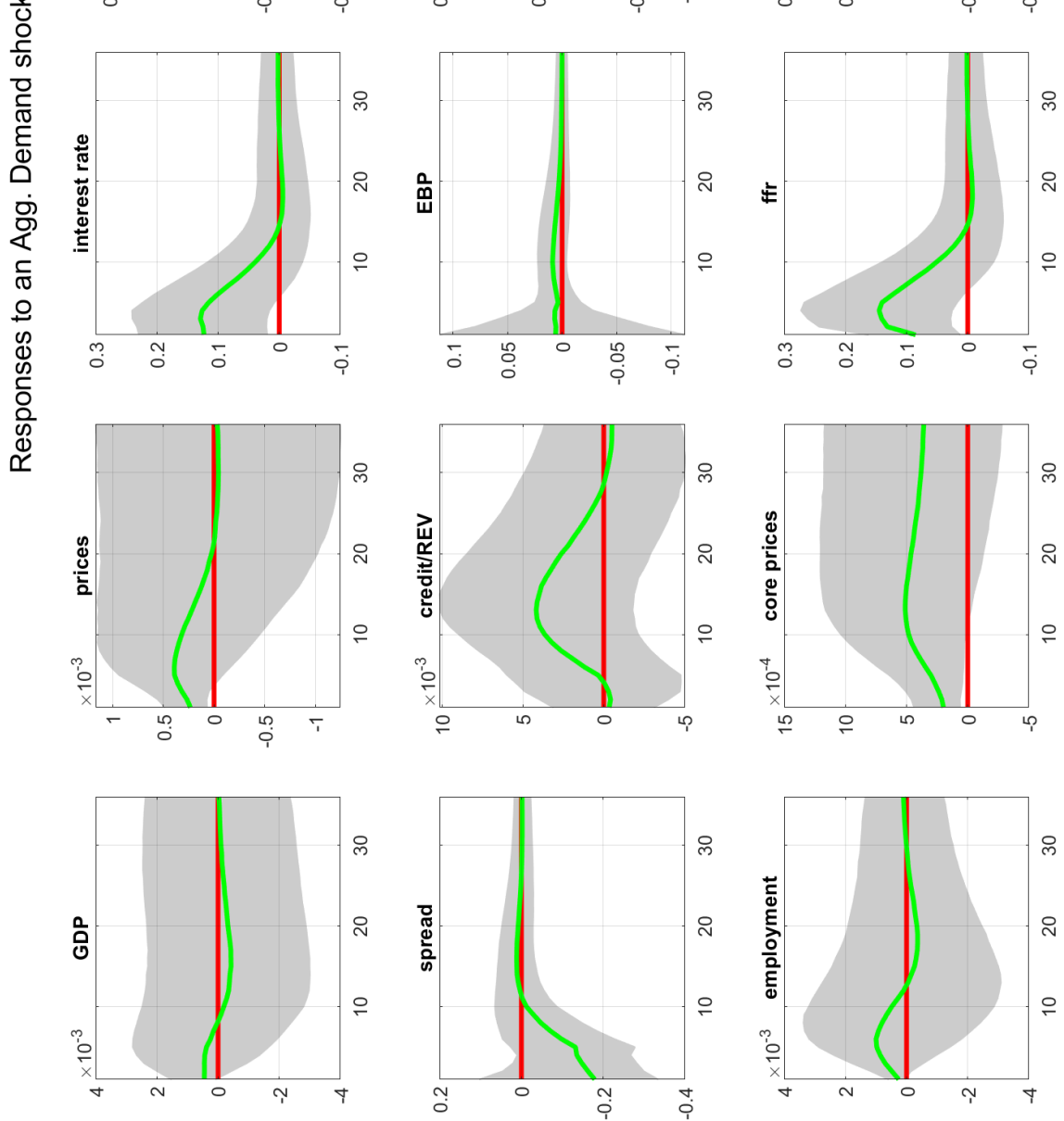

5

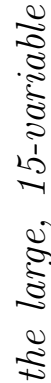

.

कृ

हี

$\frac{0}{0}$

है

हี

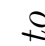

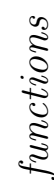

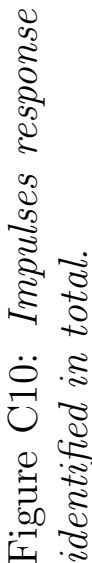



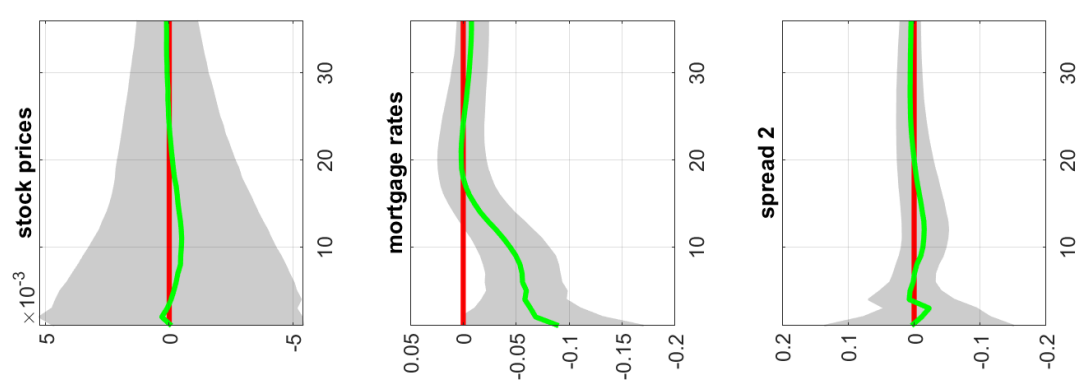

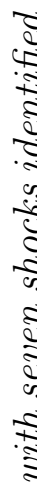
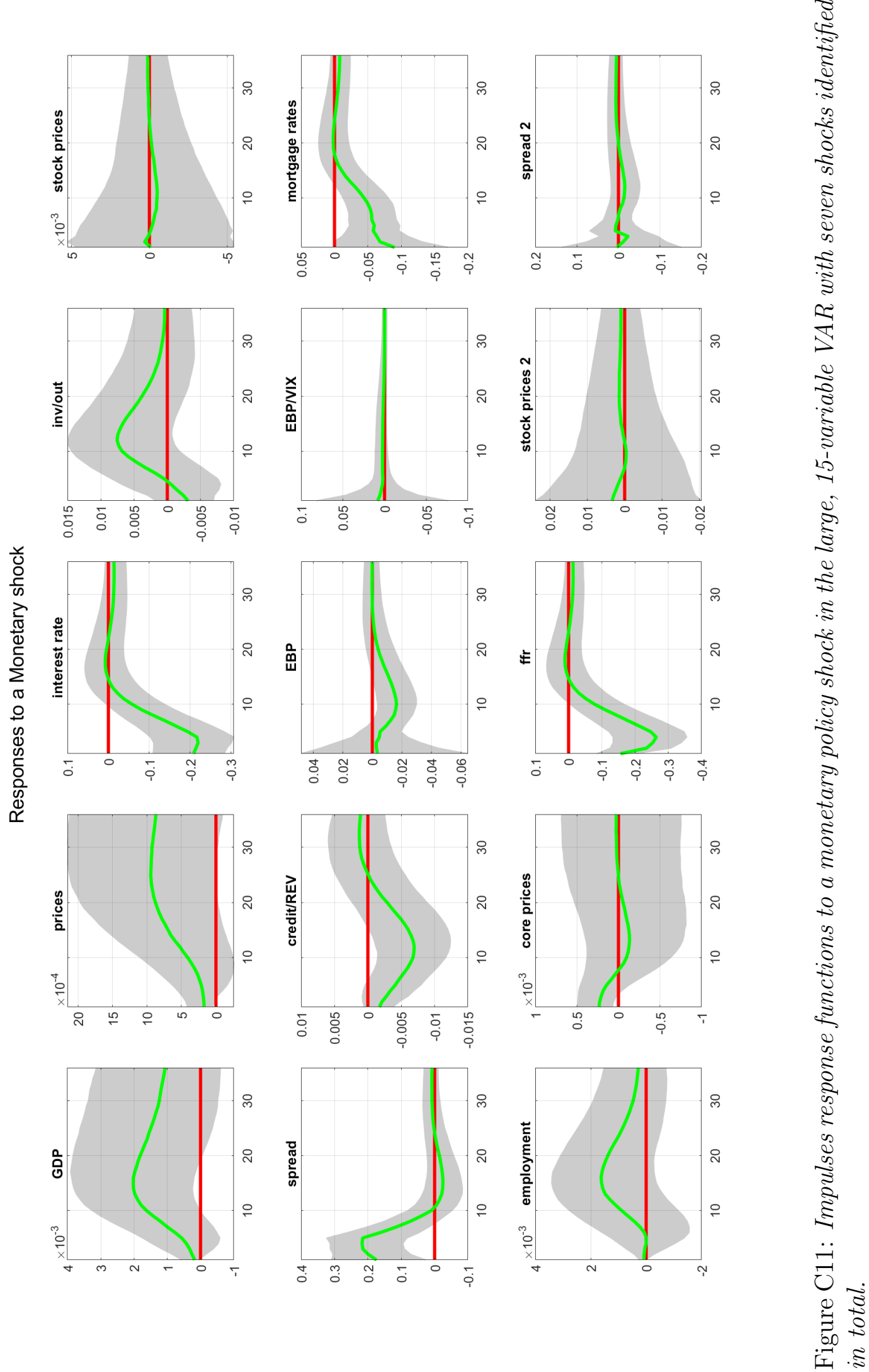

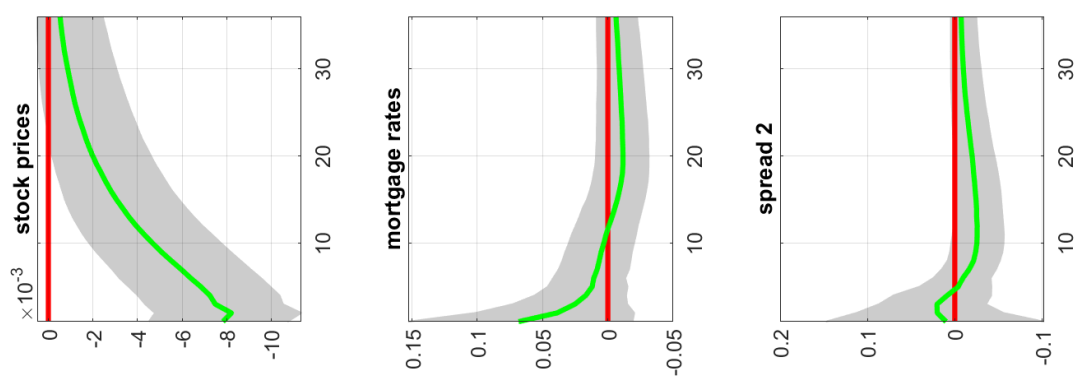

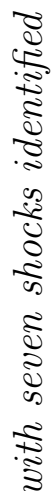
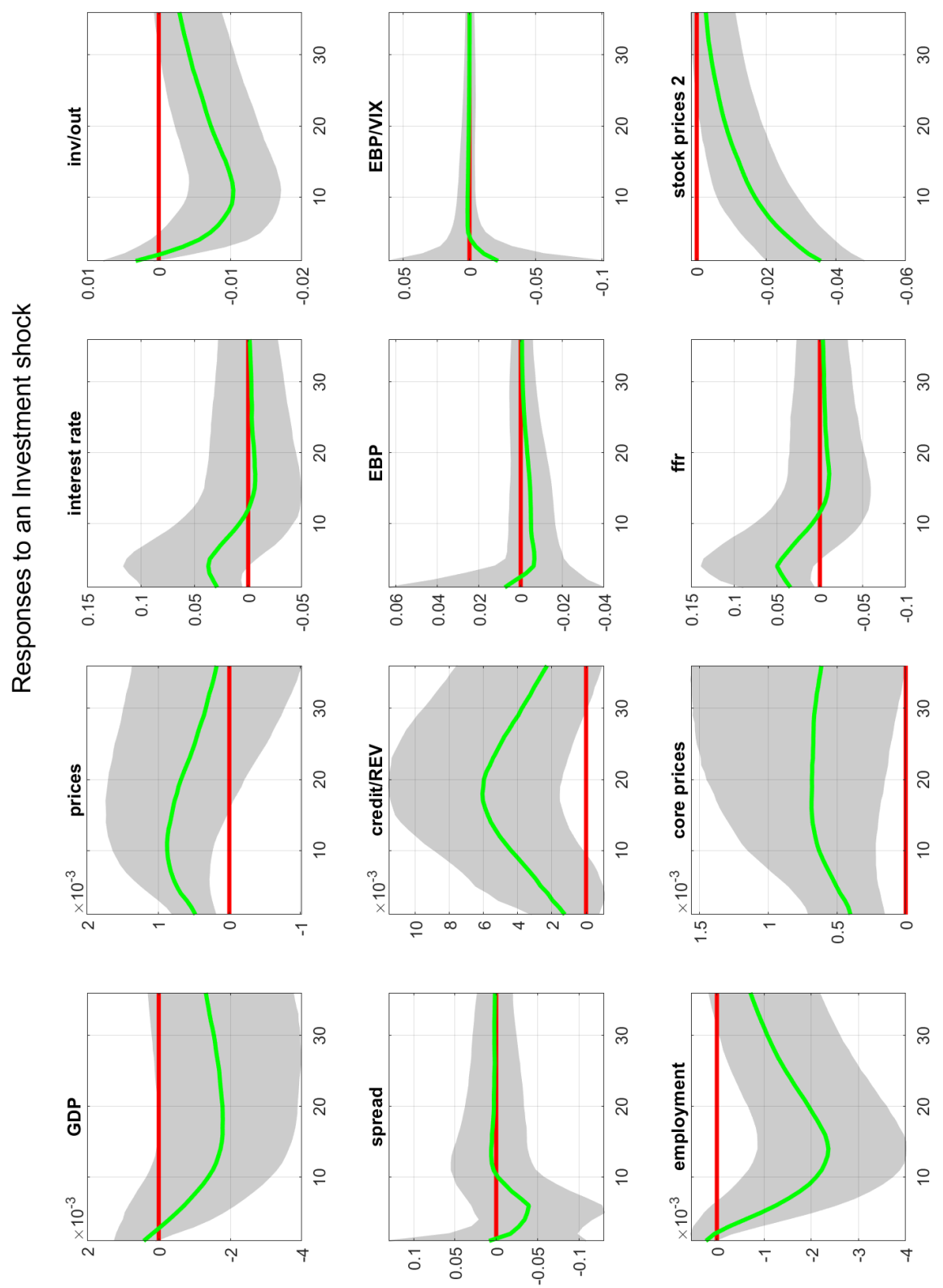

$\frac{1}{5}$

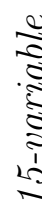

है

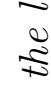

.

गु

है
है
के
हैं
है

ह

$\stackrel{ }{+}$

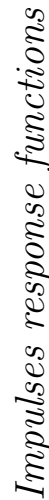

$\ddot{\ddots}$

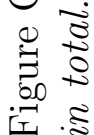



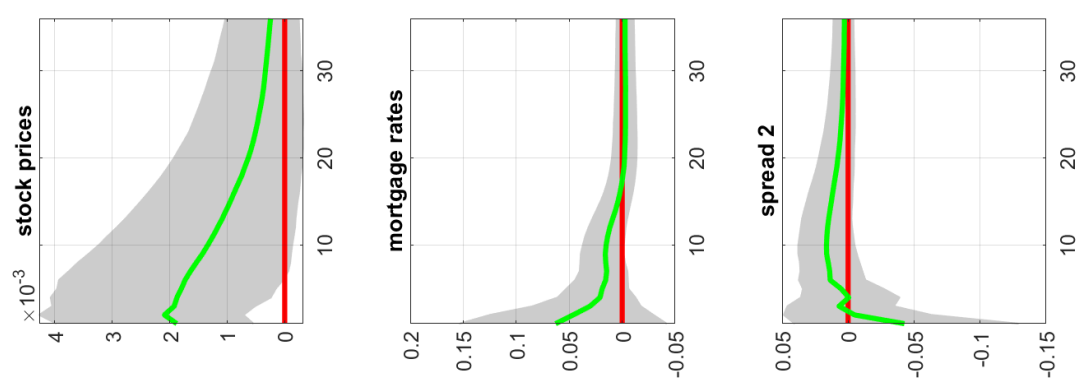

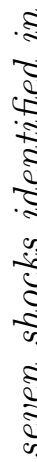
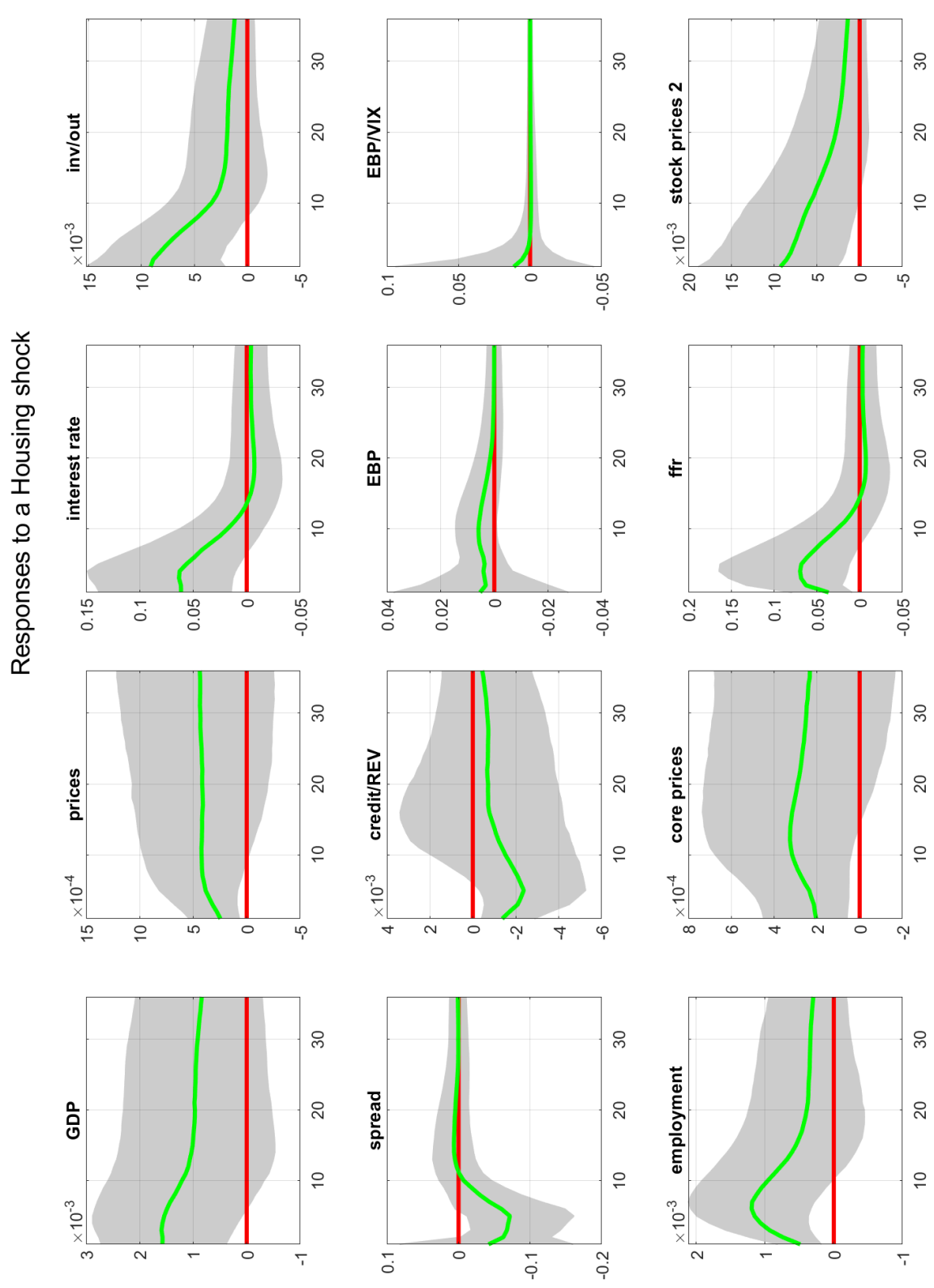

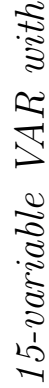

है

$\stackrel{2}{ \pm}$

.

कृ

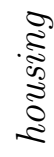

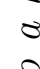

$\stackrel{0}{x}$

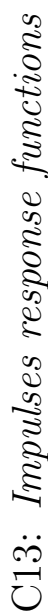

总迹 

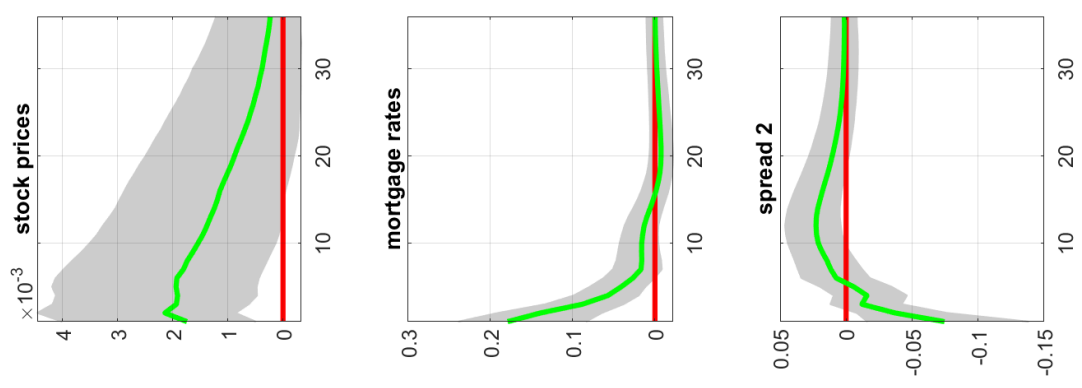

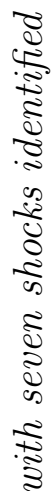
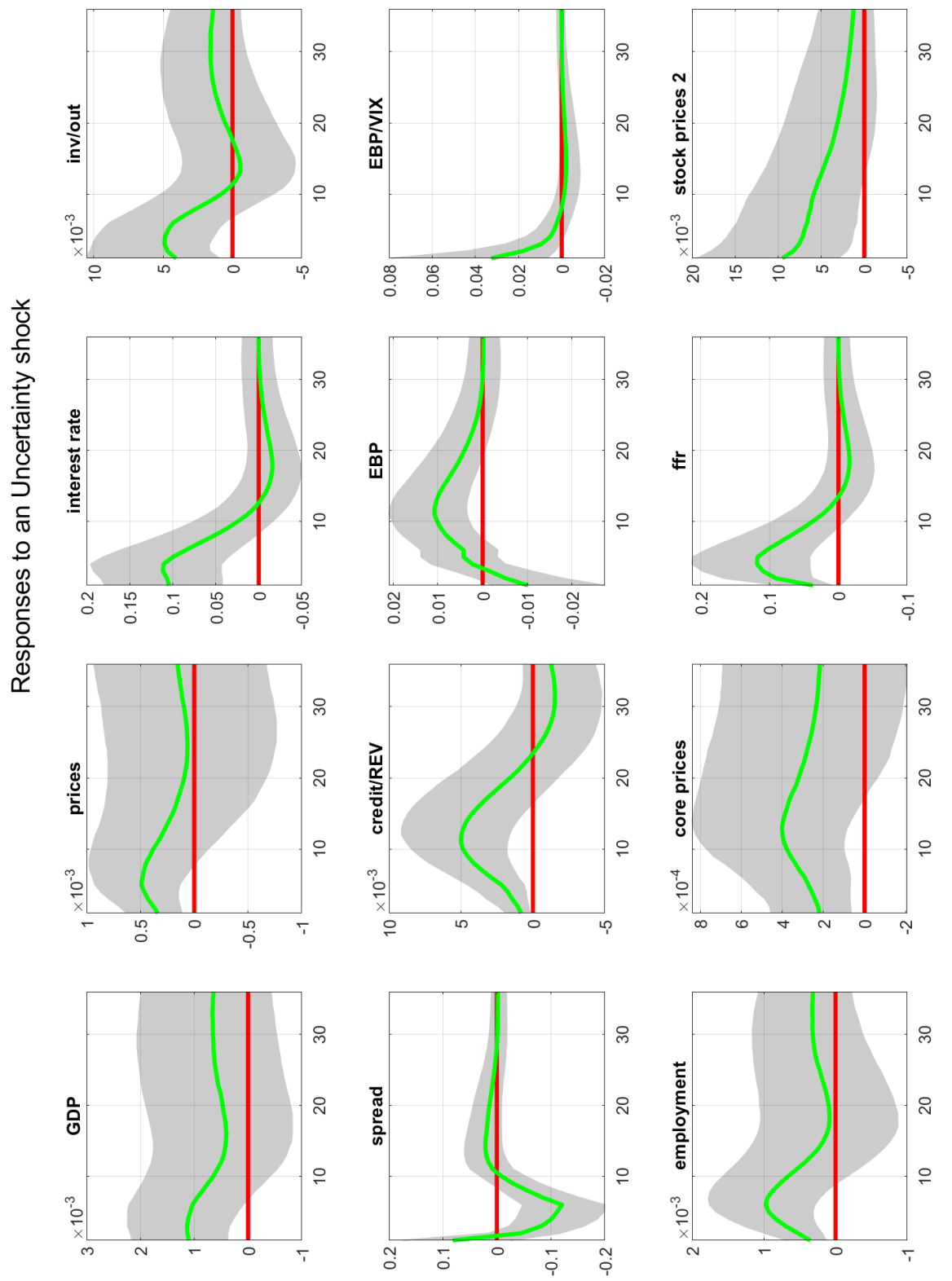

$\frac{5}{5}$

है

$\frac{0}{0}$

ล

के

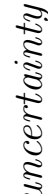

కิ

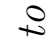

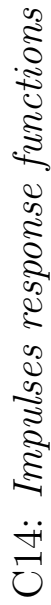

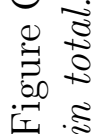

